\title{
Study on Roof-Coal Caving Characteristics with Complicated Structure by Fully Mechanized Caving Mining
}

\author{
Yunpei Liang $\mathbb{D}^{1},{ }^{1}$ Lei Li, ${ }^{1,2}$ Xuelong Li $\mathbb{D}^{1},{ }^{1}$ Kequan Wang, ${ }^{2}$ Jinhua Chen, \\ Zhongguang Sun, ${ }^{1,2}$ and Xuelin Yang $\mathbb{D}^{1,2}$ \\ ${ }^{1}$ State Key Laboratory of Coal Mine Disaster Dynamics and Control, College of Resources and Environmental Science, \\ Chongqing University, Chongqing 400044, China \\ ${ }^{2}$ National Key Laboratory of Gas Disaster Detecting, Preventing and Emergency Controlling, \\ Chongqing Research Institute of China Coal Technology and Engineering Group Crop., Chongqing 400037, China
}

Correspondence should be addressed to Xuelong Li; lixlcumt@126.com

Received 27 December 2018; Accepted 20 March 2019; Published 18 April 2019

Academic Editor: Radoslaw Zimroz

Copyright (C) 2019 Yunpei Liang et al. This is an open access article distributed under the Creative Commons Attribution License, which permits unrestricted use, distribution, and reproduction in any medium, provided the original work is properly cited.

\begin{abstract}
With mining technology and mechanization degree being improving, fully mechanized caving mining technology (FCM) has become a main method for thick coal seam extraction in China. However, roof-coal caving characteristics in turn restrict its recovery efficiency, especially for the coal seam with complicated structure (CCS), that is, the coal seam comprises hard or soft coal and gangue. In order to explore the key factors influencing the roof-coal caving and recovery characteristics, related research work has been conducted as follows: firstly, a mechanical model of CCS has been established, which indicates the strength of the coal and gangue will directly affect the roof-coal recovery. Meanwhile, based on the geological settings of Qinyuan coal mine, numerical simulation on roof-coal caving law under different thicknesses of hard or soft coal and gangue has been performed using UDEC software. The results show that the maximum principal stress will increase with the increase of mining depth, making the roof-coal to break easily. Furthermore, the range of the plastic zone of the top coal and the damage degree of the top coal increase with the increase of mining depth. Physical modeling results show that when an extraction-caving ratio is 1 , the number of times the coal arch forms is 0.43 at every caving, up to a maximum of 3 ; the number of times coal arch forms with an extractioncaving ratio of 2 is 4.65 times larger than that with an extraction-caving ratio of 1 . The probability of coal arch formation with an extraction-caving ratio of 3 is minimal, about 0.4 , which is due to that the arch span is large and the curvature is small, so it is difficult to form a stable arch structure. According to the mechanical characteristics of roof-coal in Qinyuan coal mine, deep-hole blasting technique has been used to reduce the fragments of roof-coal crushed. The results show that this technique can effectively improve the recovery of roof-coal.
\end{abstract}

\section{Introduction}

Thick coal seam $(\geq 3.5 \mathrm{~m})$ reserves account for $45 \%$ of coal reserves in China, which has great advantages in terms of resource reserves [1-4]. In recent years, with the improvement of FCM technology, it has been the main method for thick coal seam extraction [5-8]. This technique mainly involves two steps: first, a layer of coal with a thickness of $2-3 \mathrm{~m}$ at the bottom will be mined using the conventional mining method; then, under the action of mining stope pressure, the coal unmined at the high layer (roof-coal) will be fragmented and recovered by the function of self-gravity
[9-12]. As the main method for thick coal seam extraction [13-15], FCM has main advantages of high production, high efficiency, less roadway excavation, low energy consumption, etc. $[16,17]$. Therefore, it has a wide application prospect in China $[18,19]$.

Although this technique has the mentioned advantages, it also has obvious shortcoming of caving problem [20-22], which not only directly affects the difficulty of roof caving and recovery but also determines the applicability of this technology in certain geological conditions and provides key parameters for this technology and then effectively improves the roof-coal recovery [23-26]. There are major factors 
affecting roof-coal caving, including the uniaxial compressive strength $\left(\sigma_{\mathrm{c}}\right)$, the thickness of coal seam, the development of joint fractures in coal seam, etc.

The strength of coal seam mainly reflects the self-ability to resist damage, which determines the fragmentation degree of roof-coal under the action of mining stope pressure. And the fragmentation degree of roof-coal will directly affect the roof-coal recovery. During the extraction of hard coal, due to that the mining stope pressure cannot effectively fragment the roof-coal, the roof-coal blocks are too large to reach the requirement of top coal caving, which will reduce the roof-coal recovery [27-30].

Thin top coal is a pseudo-top, and it is difficult to control it from falling in the tail of the bracket, which will lead to direct crushing ahead of the top; it would mix with the top coal when released to the coal outlet, through which not only the coal quality will be affected but also a large number of top coal will be lost in the goaf; overthick top coal is hard to be fully loose in the top control area, so it is difficult to fall in the falling area. In particular, with the gradual increase in the thickness of coal seams being exploited, the mine pressure in the fully mechanized subsidence stope is becoming more intense. Whether the large-scale top coal body can be broken, fallen, or released under the action of mine pressure, the height of the fully mechanized top coal caving face can be achieved. The production rate has become a key technical problem that restricts the use of fully mechanized sublevel caving mining in extremely thick coal seams and even thick coal seams [19, 31-33].

According to on-site observations, the weakest surface of the coal affected by top coal caving in fully mechanized top coal caving face is the joints, bedding, and fractures of coal. Obviously, coal seams with joints and fissures developed have poor integrity of the coal body, and the overall strength is reduced. The top coal is easily broken under the influence of supporting pressure. At the same time, the more dense the fissures are, the more easily the top coal is broken, and the smaller the degree of eruption is, the more favorable it is to release, that is, the better the release of top coal, and vice versa [33-35]. In addition, the top coal caving is also related to the period of the roof, the depth of the coal seam, the hardness of the coal seam, the amount of sand, and the filling factor.

In recent years, with the development of science and technology, more and more advanced technological methods have been introduced into the research of fully mechanized caving mining [36]. Especially in the aspect of evaluation, many advanced calculation theories and methods are gradually introduced into the top coal evaluation. For example, the gray-fuzzy evaluation method is used to study that the steep seam coal can be relegated and fuzzy mathematics theory is used to classify the caving of top coal under different coal seam conditions [37]. Chen et al. [38] used the basic concepts of damage mechanics to describe the relationship between damage and release of top coal; Wang et al. [39] used the artificial intelligence as a reference to establish an artificial neural network process of top coal disruptive identification in steep-inclined coal caving roof caving. The above new theories and methods not only have their own characteristics but also have their certain deficiencies. For example, the fuzzy comprehensive evaluation method often requires certain subjectivity and randomness when determining the degree of membership and giving different weight to each index. The artificial neural network method has some shortcomings in slowing convergence rate, easy falling into local optimum, and hidden layer determination with subjectivity. Support vector machine to determine the boundary anti-interference ability is poor and sensitive to noise data. To this end, we also need to explore a more scientific and effective evaluation method of top coal emission. The factors influencing the runoff of top coal are complex, diverse, and nonlinear, so it is hard to come up with accurate and general discriminant criteria.

At present, fully mechanized top coal caving mining can be applied to coal seams with better geological conditions. During the fully mechanized top coal caving mining of complex structure thick coal seams, due to the presence of entrainment and hard coal, coal seams often have large fracture fragments, difficult top coal release, and poor top coal deployment. In order to study the law of breaking, releasing, and destabilization of top coal with complex structures, this paper selects the representative geology and production conditions of Qinyuan coal mine in Baoji city, China. Adopting the method of combining theoretical analysis, laboratory simulation, and numerical simulation roof breaking and releasing rules of top coal and technical parameters of caving in fully mechanized top coal caving mining with complex structure and thick coal seam, the following studies are conducted:

(1) The research results of predecessors are combined and the breaking characteristics of top coal in fully mechanized caving mining with thick seam of complicated structure are analyzed. In terms of the actual stress conditions of hard coal gangue in the fully mechanized top coal caving mining, the stability of the hard coal gangue (the hard coal delamination or the gangue in the top coal body) is studied; the mechanical model of the corresponding hardened coal gangue is established. Based on this, the crushing effect of the hardened coal gangue and the top coal caving are analyzed.

(2) The law of breaking and instability of top coal in fully mechanized top coal caving mining with complicated structure and thick seam is analyzed. $y$ means of discrete element numerical simulation method, stress field, displacement field, and distribution character of the failure field are analyzed emphatically. The crushing effect of top coal and the top coal discharge are analyzed. The law of breaking and destabilization of top coal in fully mechanized top coal caving mining with complicated structure is obtained.

(3) The simulation of laboratory bulk as a research method is performed, and the technical parameters of fully mechanized caving mining with thick coal seam with complex structure are studied. Analyze the coal gangue emanation form and top coal falling in the process of arching, and reach the complex structure of the law of the top coal emission. And put 
forward the corresponding weakening of the top coal in the hard coal gangue technical measures to improve the top coal caving.

(4) The geology and production conditions of the N101 fully mechanized caving face in Qinyuan coal mine is combined, and field measurement analysis and research is conducted to verify the research results of this paper.

\section{Mechanical Model of Complicated Structures}

Coal seams containing gangue or hard coal are often referred to as complex structural seams. According to the on-site observations and laboratory studies, it is found that, during the fully mechanized top coal caving of complex structure thick coal seams, there is a large difference in the top coal brittleness, which directly leads to the phenomenon that top coal is often difficult to emerge during top coal caving. Therefore, the mechanical model of hard coal stratification or vermiculite layer in top coal is established. On the one hand, it can give quantitative explanation of the breaking degree and release of complex structure top coal; on the other hand, it can be used to solve engineering problems (top coal breakage, characteristics of caving and drawing problems, etc.) and propose engineering measures based on theoretical basis.

\subsection{Deformation Characteristics of Top Coal in Complex} Structures. Regardless of whether the top coal contains holding gangue or hard top coal stratification, as the thickness of the hard strata coal roof in the top coal increases, the overall strength of the top coal increases, and the fracture characteristics of the medium top coal or hard top coal are gradually shown. After the coal body deformation exceeds the peak, the top coal enters the plastic deformation state, and the hard coal gangue may only enter the strength destruction stage. With the development of the damage, the stability of the plastic zone is reduced until the instability. The length of destabilization depends on the extent of the plastic zone in front of the coal wall, the length of the top beam of the support, and the advancing speed of the working face. The strength of the coal gangue is low, and the granular area of the top coal extends to the front of the coal wall, which is unfavorable for the maintenance of the end face. The strength of the coal gangue is high, and there is little part of the top coal in the granular region at the rear of the support; that is, there are few broken blocks and particles, and the top coal is very low. It is usually necessary to weaken the hard coal gangue in the top coal, such as deep-hole blasting and top coal water injection to improve the characteristics of top coal caving and drawing in fully mechanized top coal caving mining. In the cavities above the support, the vertical displacement of the top coal is greater than the horizontal displacement. The displacement of the low-strength hard coal gangue is much larger than that of the high-strength hard coal ram, which indirectly reflects the phenomenon that the low-strength coal gangue is broken into granules and the high-hardness coal gangue is broken into blocks. The top coal emission is different.
It is known that the difference in the strength of the hard coal gangue makes the top coal to have different elastic-plastic zoning, resulting in a great difference in the top coal broken block [7, 24]. The top coal at the coal seam front of the complex structure coal seam is in the triaxial stress state, and the top coal deformation is dominated by the horizontal deformation. For the top coal with high strength and large thickness of hard coal gangue, the crack development of the top coal is relatively weaker than the strength and thickness, while the top coal in the top control area is dominated by the vertical displacement. The top coal with hard-bed coal gangue is not developed in the bedding and the weak side, resulting in difficulty in delamination. The vertical displacement of the top coal is lower than that of the hard coal gangue top coal with low strength, and the deformation is not obvious. Besides, the coal is not fully broken, and the high-strength and thick-layer top coal breaks a large block, causing difficulty in discharge, and even blocking the coal opening.

\subsection{Stiffness Characteristics of Top Coal in Complicated} Structures. The damage of the top coal after reaching the peak strength in a complex structure depends on the degree of the top coal deformation, and the size of the top coal deformation is determined by the stiffness of the hard coal and top coal. To simplify the problem, it is assumed that the stiffness of the bracket and the direct top is considered to be large, irrespective of the influence of the bracket and the direct top (Figure 1). Then, the stiffness $K_{\mathrm{m}}$ of the hard layer coal gangue $K_{\mathrm{c}}$ and the top coal is analyzed.

Assuming that the force in the vertical direction of hard coal gangue and top coal is $F$ and the total compression of top coal is $\Delta S$, the total top coal stiffness is

$$
K=\frac{1}{\left(1 / K_{\mathrm{c}}\right)+\left(1 / K_{\mathrm{m}}\right)}=\frac{K_{\mathrm{c}} \cdot K_{\mathrm{m}}}{K_{\mathrm{c}}+K_{\mathrm{m}}} .
$$

Then,

$$
F=K \cdot \Delta S=\frac{K_{\mathrm{c}} \cdot K_{\mathrm{m}}}{K_{\mathrm{c}}+K_{\mathrm{m}}} \cdot \Delta S=K_{\mathrm{m}} \cdot \Delta S_{\mathrm{m}} .
$$

Through calculation, the compression of hard coal gangue is $\Delta S_{\mathrm{m}}=\left(K_{\mathrm{c}} / K_{\mathrm{c}}+K_{\mathrm{m}}\right) \cdot \Delta S$.

That is,

$$
\frac{\Delta S_{\mathrm{m}}}{\Delta S}=\frac{K_{\mathrm{c}}}{K_{\mathrm{c}}+K_{\mathrm{m}}}=\frac{\left(K_{\mathrm{c}} / K_{\mathrm{m}}\right)}{\left(K_{\mathrm{c}} / K_{\mathrm{m}}\right)+1} .
$$

From equation (3), it can be seen that the percentage of top coal deformation increases with the increase in $K_{\mathrm{c}} / K_{\mathrm{m}}$. When the stiffness $K_{\mathrm{c}}$ of hard coal gangue is less than the top coal stiffness $K_{\mathrm{m}}$, the top coal produces less deformation, while the hard coal tar results in greater deformation, which is beneficial for the cracking of the hard layer coal gangue and the improvement of characteristics of top coal caving and drawing. When the top coal stiffness $K_{\mathrm{m}}$ is less than the hard layer coal enthalpy stiffness $K_{\mathrm{c}}$, the hard layer coal tarpaulin will produce less deformation, the old top and direct top rotatory deformation will be absorbed by the top coal, and the top coal will be broken before the hard layer coal gangue. Broken blocks block the coal open or make it 


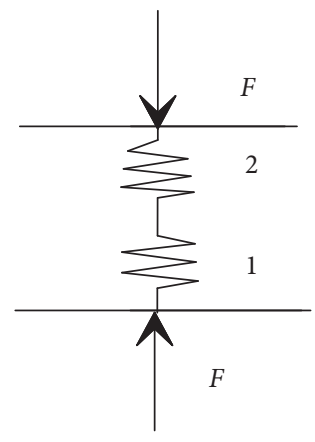

Figure 1: Stiffness of the series system between hard coal gangue and top coal. (1) Top coal stiffness $K_{\mathrm{m}}$. (2) Hard layer coal gangue stiffness $K_{\mathrm{c}}$.

difficult to break the hard coal gangue, reducing the overall top coal deployment. The comparison of the top coal stiffness $K_{\mathrm{m}}$ and the stiffness $K_{\mathrm{c}}$ of the hard coal rake has a significant effect on the fracture of the top coal in complex structures.

\subsection{Stability Analysis of Top Coal in Complex Structures.} The majority of rock formations in coal mines are sedimentary rocks. After mining, most of the surrounding rock in the mining area is dominated by gravity stress fields. The following is a mechanical analysis of the hard coal gangue in the top coal in the self-weight stress field.

The hard-coal coal gangue in the top coal outside the peak area of support pressure ahead of the complex coal seam coal wall is basically in the elastic stage. That is, the hard coal gangue is located in the peak area of the support pressure or in the top control zone. Due to the high strength and hardness of the hard layer coal gangue, with the increase of the top coal load above the support, the top coal enters the plastic state under the support pressure, which acts as a cushion for the hard layer coal gangue and makes the top coal relatively complete. Therefore, the hard coal gangue is still in the elastic stage. In the hard coal gangue, remove the tiny unit, and it can be treated as an elastic medium; according to the physical equation of the space stress state,

$$
\varepsilon_{3}=\frac{1}{E}\left[\sigma_{3}-\mu\left(\sigma_{1}-\sigma_{2}\right)\right] \text {. }
$$

If $\sigma_{2}=\sigma_{3}$, then

$$
\varepsilon_{3}=\frac{1}{E}\left[(1-\mu) \sigma_{3}-\mu \sigma_{1}\right] .
$$

Also know

$$
\varepsilon_{3}=-\mu \frac{\sigma_{c}}{E}
$$

Substituting (5) into (6):

$$
-\mu \frac{\sigma_{3}}{E}=\frac{1}{E}\left[(1-\mu) \sigma_{3}-\mu \sigma_{1}\right] .
$$

When finishing the hard layer coal gangue medium to reach the ultimate state of damage, the elastic fracture stress formula is as follows:

$$
\sigma_{1}=\sigma_{\mathrm{c}}+\frac{1-\mu}{\mu} \sigma_{3},
$$

where $\sigma_{1}$ and $\sigma_{3}$ are the vertical and lateral stresses of hard coal gangue, respectively; $\sigma_{\mathrm{c}}$ is the uniaxial compressive strength of the hard coal gangue; and $\mu$ is Poisson's ratio of the hard coal gangue. From equation (8), it can be seen that the elastic fracture stress value of hard coal gangue increases with the increase in unidirectional compressive strength and lateral stress. The formula can be translated into

$$
\sigma_{1}=\sigma_{\mathrm{c}}=\left(\frac{1}{\mu}-1\right) \sigma_{3} .
$$

Similarly, the formula for the elastic fracture stress at the top stage of the coal body in front of the coal wall is

$$
\sigma_{1}^{\prime}=\sigma_{\mathrm{c}}^{\prime}=\left(\frac{1}{\mu^{\prime}}-1\right) \sigma_{3}^{\prime}
$$

where $\sigma_{1}^{\prime}$ and $\sigma_{3}^{\prime}$ are the top coal vertical and lateral stresses, respectively; $\sigma_{\mathrm{c}}^{\prime}$ is the unidirectional compressive strength of the top coal; and $\mu^{\prime}$ is Poisson's ratio of the top coal.

In the case that the hard coal gangue is combined with actual field data, the values are $\sigma_{\mathrm{c}}=35 \mathrm{MPa}, \mu=0.3, K=3$, $\gamma=2500 \mathrm{kN} / \mathrm{m}^{3}$, and $H=520 \mathrm{~m}$. Through calculation, $K \gamma H=39 \mathrm{MPa}$ and $\sigma_{1}=41 \mathrm{MPa}$.

From the data calculation under hard coal conditions, it can be inferred that, under the effect of mining stress, the abutment pressure peak is greater than the numerical value of the elastic fracture stress of hard coal gangue. The crack density of the hard coal gangue will not expand and the stress value of the block broken in advance will not be reached.

The soft coal above the hard coal gangue is also taken as $\sigma_{\mathrm{c}}^{\prime}=8 \mathrm{MPa}, \mu^{\prime}=0.4, K=3, \gamma=2500 \mathrm{kN} / \mathrm{m}^{3}$, and $H=520 \mathrm{~m}$. Through calculation, $K \gamma H=39 \mathrm{MPa}$ and $\sigma_{1}^{\prime}=34 \mathrm{MPa}$.

From the calculation of data under soft coal conditions, we can see $K \gamma H>\sigma_{1}^{\prime}$; that is, when the peak bearing pressure is greater than the soft coal elastic fracture stress value, the crack density of the hard coal gangue expands and penetrates and the overall strength decreases, breaking into irregular blocks in advance.

From the analysis, it can be known that whether the top coal in a complex structure contains a clamp or a hard top coal, the elastic fracture stress value of a hard coal seam is usually greater than the elastic fracture stress value of the top coal. That is, $\sigma_{1}-\sigma_{1}^{\prime}>0$.

The comparison between the peak pressure of support pressure and the elastic rupture stress value of soft coal gangue shows whether the hard coal gangue is cracked or not, and it is difficult to describe the degree of rupture. The uniaxial compressive strength values of hard coal gangue and soft coal are fixed, and the concept of rupture factor is introduced, namely, the ratio of the peak value of the leading support pressure and the uniaxial compressive strength. The value is

$$
\begin{array}{r}
i=\frac{K \gamma H}{\sigma_{\mathrm{c}}}, \\
i^{\prime}=\frac{K \gamma H}{\sigma_{\mathrm{c}}^{\prime}},
\end{array}
$$

where $i$ is the hard coal failure factor and $i^{\prime}$ is the soft coal fracture factor. 
When the support pressure peak value $K \gamma H$ is $39 \mathrm{MPa}$, the hard coal in the hard coal slag is selected as the representative study. The uniaxial compressive strength $\sigma_{c}$, the hard coal fracture coefficient $i$, and the soft coal fracture coefficient $i^{\prime}$ are shown in Table 1.

As can be seen from Figure 2, the hard coal failure coefficient $i$ and the soft coal rupture factor $i^{\prime}$ are strongly related to their respective compressive strength values and are exponentially related. While hard coal, usually $\sigma_{\mathrm{c}} \geq 30 \mathrm{MPa}$; when $\sigma_{\mathrm{c}} \geq 30 \mathrm{MPa}$, the hard coal failure factor $i$ in the hard coal gangue has a maximum of 1.3. That is, as the value of the uniaxial compressive strength of hard coal increases, $i$ decreases, and the closer to the maximum, the larger the $i$, the better the cracking effect of the hard coal. While soft coal, usually $\sigma_{\mathrm{c}} \leq 10 \mathrm{MPa}$; when $\sigma_{\mathrm{c}}=10 \mathrm{MPa}$, the soft coal fracture coefficient $i^{\prime}$ has a minimum value 3.9; that is, as the uniaxial compressive strength of soft coal decreases, $i^{\prime}$ increases, and the farther away from the minimum, the better the soft coal crushing effect.

Top coal working face stress concentration coefficient $K$ is generally taken from 2 to 3 . Similarly, it can be concluded that when the stress concentration factor is taken from other values in the same depth, the hard coal fracture coefficient $i$ and the soft coal rupture factor $i^{\prime}$ have the same linear correlation with their respective compressive strength values, and they also have an exponential function relationship. The larger the fracture coefficient $i$, the better the crushing effect of the hard layer coal gangue and the release of the top coal; the larger the crumple factor $i^{\prime}$, the better the crushing effect of the soft top coal and the characteristics of top coal caving and drawing.

Under the certain conditions of the leading bearing pressure peak $K \gamma H$, the soft coal fracture coefficient $i^{\prime}$ is a fixed value, and the smaller the value of $i^{\prime}-i$, the smaller the difference in the breaking block of hard coal and soft coal, and the better the crushing effect. Both have gone through the process of fracture development and penetration, and then through the deformation of the top of controlled top area, the entire change process of the mechanics shows that there is little difference between the breaking of the hard coal gangue and the top coal. The hard layer coal gangue rupture coefficient $i$ is a fixed value. The larger the value of $i^{\prime}-i$, the smaller the difference in the breaking layer degree between the hard layer coal gangue and the soft coal, the better the crushing effect, otherwise the difference in the broken lumping degree is large, and the top coal is spread out poorly. When the hard coal gangue breaking block is larger, the arch structure is easily formed during the dropping process and the coal opening, which hinders the normal flow of the top coal. Due to the large difference in the block size, the flow rate is not balanced. It is disadvantageous to flow and recovery of the top coal.

\section{Numerical Simulation Study}

The discrete element method was firstly proposed by Cundall. P. A. in 1971 as a discontinuous medium numerical analysis method. It can both simulate the movement of the block after the force and simulate the deformation state of the block itself.
TABLE 1: Hard coal failure factor $i$ and soft coal failure factor $i^{\prime}$ in hard coal gangue.

\begin{tabular}{lccccccc}
\hline$\sigma_{\mathrm{c}}$ & 30 & 35 & 40 & 45 & 50 & 55 & 60 \\
$i$ & 1.3 & 1.11 & 0.98 & 0.87 & 0.78 & 0.71 & 0.65 \\
$\sigma_{\mathrm{c}}^{\prime}$ & 10 & 8 & 7 & 6 & 5 & 4 & 3 \\
$i^{\prime}$ & 3.9 & 4.88 & 5.57 & 6.5 & 7.8 & 9.75 & 13 \\
\hline
\end{tabular}

In this paper, the numerical simulation program UDEC is used to simulate the stress, strain, and displacement of the top coal during the fully mechanized top coal caving mining in the thick seam with complex structure. Its advantages lie in the fact that firstly, discrete rock masses allow large deformations, allowing sliding along joint surfaces, turning and falling out of the joints, and secondly, new contacts can be automatically identified during the calculation process.

3.1. Model Establishment. According to the geological conditions of the N101 fully mechanized caving face of Qinyuan coal mine, the model size is $220 \mathrm{~m} \times 49.8 \mathrm{~m}$, and the upper boundary load is calculated by the depth of $520 \mathrm{~m}$. The direct top thickness is $1.6 \mathrm{~m}$, the block size is $1.6 \mathrm{~m} \times 0.8 \mathrm{~m}$, and the old top is $12 \mathrm{~m}$ thick. Breaking step distance $16 \mathrm{~m}$. Direct bottom thickness $1.5 \mathrm{~m}$, length $3 \mathrm{~m}$; old bottom thickness $5 \mathrm{~m}$, block size $2.5 \mathrm{~m} \times 5 \mathrm{~m}$. The simulated coal seam thickness is $8.7 \mathrm{~m}$, of which the mining height is $2.3 \mathrm{~m}$ and the coal laying height is $6.4 \mathrm{~m}$. Because the object of simulation analysis is complex structure top coal, in order to improve the calculation accuracy, the top coal in the middle part of the model is encrypted, the top coal contains a hard layer coal gangue, and the model divides the hard coal gangue in the coal seam. For the upper, middle, and lower cases, the top coal in the fully mechanized top coal caving face was simulated to be fractured and unstable. Here, the hard coal gangue is regarded as a hard continuous medium. The control resistance of the top control zone is constant resistance, and the support control distance is $4 \mathrm{~m}$.

3.1.1. Determination of the Model Geometry. The top coal is soft coal. When the thickness of the hard coal gangue is $1.5 \mathrm{~m}$, the divisional degree is $1.5 \mathrm{~m} \times 0.9 \mathrm{~m}$, and the top coal is divided into $0.83 \mathrm{~m} \times 0.42 \mathrm{~m}$. When the thickness of the hard coal gangue is $0.5 \mathrm{~m}$, the division degree is $0.5 \mathrm{~m} \times 0.3 \mathrm{~m}$, and the top coal is divided into blocks of $0.98 \mathrm{~m} \times 0.49 \mathrm{~m}$. In addition, some numerical simulation conditions or parameters in the model are analyzed in order to analyze the failure of the top coal in the complex structure coal seam under conditions of different strengths and different mining depths ( $H=320 \mathrm{~m}, H=520 \mathrm{~m}$, and $H=720 \mathrm{~m})$ of the hard coal gangue. Adjustments were made to simulate the stress distribution characteristics, displacement distribution characteristics, and failure characteristics of top coal under different conditions in top coal.

3.1.2. Selection of Rock Mechanics Parameters. According to the geological conditions of the actual working face, the surrounding rock mechanics parameters are selected, as shown in Tables 2 and 3. 


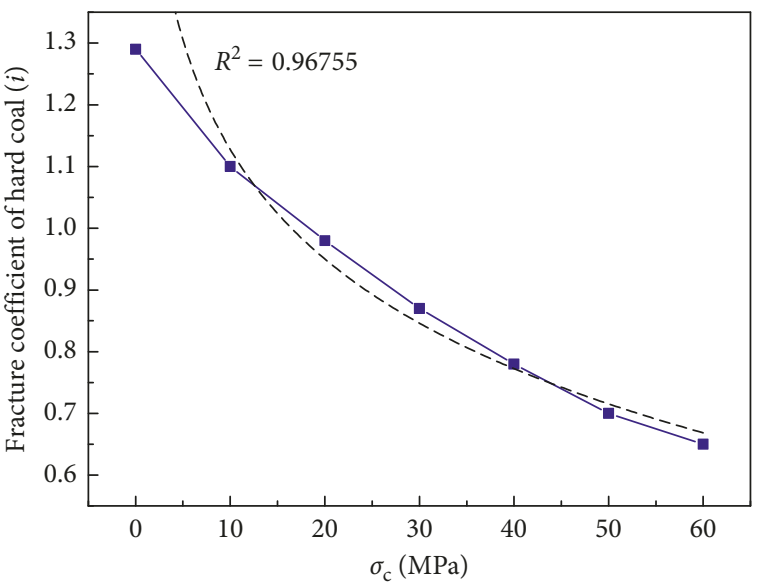

(a)

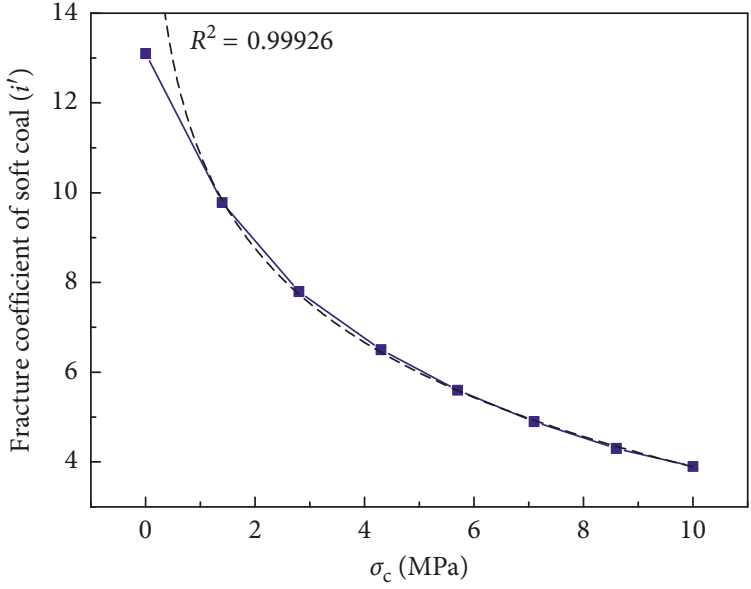

(b)

FIGURE 2: Relationship between function coefficients of hard and soft coal. (a) Hard coal failure factor (i) function in hard coal gangue. (b) Soft coal fracture factor $\left(i^{\prime}\right)$ function.

TABLE 2: Mechanical parameters of hard layer coal gangue in different layers of top coal.

\begin{tabular}{|c|c|c|c|c|c|c|}
\hline Rock stratum & $\begin{array}{c}\text { Density, } \\
d\left(\mathrm{~N} \cdot \mathrm{m}^{-3}\right)\end{array}$ & $\begin{array}{c}\text { Bulk modulus, } \\
K(\mathrm{GPa})\end{array}$ & $\begin{array}{c}\text { Shear modulus, } \\
G(\mathrm{GPa})\end{array}$ & $\begin{array}{c}\text { Internal friction } \\
\text { angle, } f\left({ }^{\circ}\right)\end{array}$ & $\begin{array}{c}\text { Adhesion, } \\
C(\mathrm{MPa})\end{array}$ & $\begin{array}{c}\text { Tensile strength, } \\
t(\mathrm{MPa})\end{array}$ \\
\hline Hard coal gangue (hard coal) & 1600 & 12 & 10 & 34 & 4 & 2 \\
\hline Hard coal gangue (gangue) & 2600 & 18 & 14 & 36 & 19 & 12 \\
\hline Top coal & 1300 & 5 & 2.2 & 21 & 1 & 0.8 \\
\hline Overlying strata & 2700 & 20 & 17 & 42 & 20 & 11 \\
\hline Basic roof & 2700 & 20 & 17 & 42 & 20 & 11 \\
\hline Immediate roof & 2500 & 13 & 10 & 38 & 10 & 4 \\
\hline False roof & 2100 & 13 & 7 & 36 & 8 & 3 \\
\hline Direct bottom & 1800 & 10 & 10 & 32 & 5 & 3 \\
\hline Previous bottom & 2200 & 19 & 16 & 40 & 7 & 4 \\
\hline
\end{tabular}

TAвLe 3: Mechanical parameters of contact surface between hard seam and coal seam in different layers of top coal.

\begin{tabular}{lccccc}
\hline Rock stratum & $\begin{array}{c}\text { Normal stiffness, } \\
\mathrm{jkn}(\mathrm{GPa})\end{array}$ & $\begin{array}{c}\text { Shear stiffness, } \\
\mathrm{jks}(\mathrm{GPa})\end{array}$ & $\begin{array}{c}\text { Internal friction } \\
\text { angle, } f\left(^{\circ}\right)\end{array}$ & $\begin{array}{c}\text { Cohesive force, } \\
C(\mathrm{MPa})\end{array}$ & $\begin{array}{c}\text { Tensile strength, } \\
t(\mathrm{MPa})\end{array}$ \\
\hline Hard coal gangue (hard coal) & 4 & 3.5 & 27 & 0.04 & 0 \\
Hard coal gangue (gangue) & 14 & 5 & 29 & 0.05 & 0.02 \\
Top coal & 4 & 3 & 3 & 0.07 & 0 \\
Overlying strata & 16 & 6 & 31 & 0.07 & 0 \\
Basic roof & 16 & 6 & 0 & 0 & 0 \\
Immediate roof & 7 & 5 & 0 & 0.06 & 0 \\
False roof & 6 & 4.5 & 28 & 0.1 & 0 \\
Direct bottom & 5 & 6 & 32 & 0 \\
Previous bottom & 5 & 6 & & 0 \\
\hline
\end{tabular}

3.1.3. Determination of the Boundary Conditions. According to the actual occurrence conditions of the calculation model, the boundary conditions of this calculation model are as follows.

Upper boundary condition: This is related to the overburden gravity $\left(\sum \gamma h\right)$. In order to facilitate the study, the distribution of the load is simplified as a uniform load. The upper boundary condition is the stress boundary condition:

$$
q=\sum \gamma h=13 \mathrm{MPa}
$$

Lower boundary conditions: the lower boundary condition of this model is the bottom plate, which is simplified as a displacement boundary condition. It can move in the $x$ direction, and the $y$ direction is a fixed hinge support, that is, $v=0$.

Boundary conditions on both sides: the boundary conditions on both sides of this model are solid coal rock 
bodies, which are simplified as displacement boundary conditions and can move in the $y$ direction. The $x$ direction is a fixed hinge support: $u=0$.

\subsection{Simulation Results and Analysis}

3.2.1. Stress Field Analysis. Figure 3 reflects the vertical stress distribution in front of the coal wall with different thicknesses and hard coal seams. From this, it can be seen that the vertical stress has played a role in destroying the top coal of the complex structure, and this determines the degree of fracture when the top coal reaches the top of the coal wall. The vertical stress of $7 \mathrm{~m} \sim 16 \mathrm{~m}$ in front of the $1.5 \mathrm{~m}$ thick hard-coal coal gangue coal wall is in the peak position, that is, the peak support pressure area. The vertical stress increases quickly from $16 \mathrm{~m}$ to $24 \mathrm{~m}$ in front of the coal wall, and the vertical stress gradually goes to the original rock stress at $24 \mathrm{~m}$. The vertical stress at $18 \mathrm{~m}$ in front of the $0.5 \mathrm{~m}$ thick hard coal gangue coal gangue reaches the peak point, and the vertical stress is less than the $1.5 \mathrm{~m}$ thick vertical boring coal gangue stress value. The peak zone is $0.5 \mathrm{~m}$ thick, and hard coal gangue is flat and far from the coal wall. It can be seen that the supporting pressure of the $1.5 \mathrm{~m}$ thick hard coal gangue shows the distribution characteristics of the abutment pressure of the hard top coal, and the supporting pressure of the $0.5 \mathrm{~m}$ thick hard tar coal gangue shows the distribution characteristics of the abutment pressure of the hard top coal.

Figure 4 shows the distribution of shear stress in different layers of coal seams with $1.5 \mathrm{~m}$ thickness and different strengths. From the analysis and comparison in Figure 5, the maximum shear stress value of the $1.5 \mathrm{~m}$ thick medium-hard layer coal gangue is higher than the maximum shear strength value of $4.58 \mathrm{MPa}$, and the breaking effect of hard coal gangue and top coal is good, and the high-strength coal gangue develops the shear stress. Basically, the pressureshear stress occurs, and the tensile shear stress region is small. The tensile shear stress and the compressive shear stress of the medium-strength hard coal gangue exist.

Figure 5 shows the maximum and minimum principal stress difference curves for different layers of $1.5 \mathrm{~m}$ thick medium-strength hard-coal coal gangue. Shear stress can be expressed as a function of the relationship between the maximum principal stress and the minimum principal stress. The strength of the top coal and hard layer coal gangue in the peak support pressure area continuously decreases. When the hard coal gangue is in the lower part of the top coal, the main stress difference is the largest. At this time, the hard coal gangue is affected by the large shear layer coal gangue. The main stress difference is the largest. That is, the shear fracture occurs, the principal stress (maximum principal stress and minimum principal stress) in the control top area reduces rapidly, the overall rigidity also reduces, the breaking block is small, and the breaking effect is good, which make the top coal to fall smoothly. When the hard coal gangue is in the middle and upper part of the top coal, the variation of the principal stress difference is lower than that in the lower. The hard coal gangue has a poor breaking

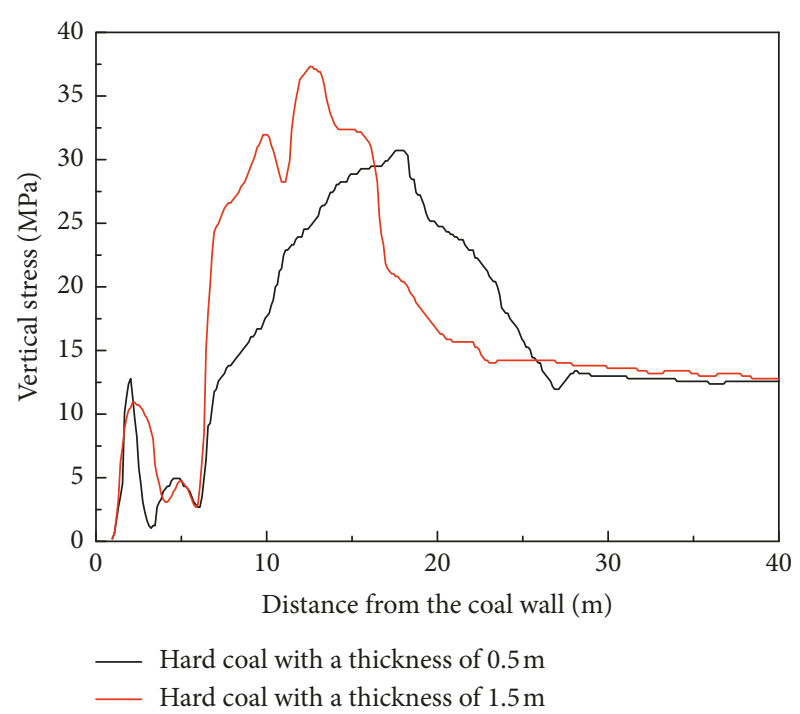

Figure 3: Vertical stress of the coal body in front of coal wall with different thicknesses of hard coal seam.

effect, a large block size, or a cantilever structure, resulting in poor top coal deployment.

As shown in Figure 6(a), the maximum principal stress of the top coal in the $1.5 \mathrm{~m}$ thick medium-hard layer coal gangue with depth $H=320 \mathrm{~m}$ is $8 \mathrm{~m}$ away from the coal wall. When the burial depth is $H=520 \mathrm{~m}$, the maximum principal stress of the top coal is $6 \mathrm{~m}$ away from the coal wall, and when the burial depth is $H=720 \mathrm{~m}$, the maximum principal stress of the top coal is $11 \mathrm{~m}$ away from the coal wall. With the increase in the depth of the top coal, the maximum principal stress value also increases, but the plastic mobility of the top coal increases, the bearing capacity decreases, and the maximum principal stress decreases with the depth $H=720 \mathrm{~m}$.

As shown in Figure 6(b), with the increase of the depth of the top coal, the maximum principal stress value also increases. Under the condition of burial depth of $H=720 \mathrm{~m}$, the top coal in the area of 8 to $10 \mathrm{~m}$ in front of the coal wall cannot withstand the old top. The slewing pressure reduces the maximum principal stress.

3.2.2. Displacement Field Analysis. In the case of upper hard coal gangue, the subsidence rate of the top coal with small breaking block is faster than that of the hard layer coal gangue with large breaking fragment, while the top coal block reaches the coal before the hard coal lumping block (Figure 7). There has no effect on the release of top coal. In the middle hard layer coal gangue condition, the top coal below the hard coal gangue is smoothly and quickly released with the shift frame, the middle hard layer coal gangue forms the delamination, and the intermediate hard gangue coal gangue breaks insufficiently and breaks the fragmentation degree big. Because the lower speed of the hard coal gangue block is slower than that of the top coal, it will hinder the top coal body, and the top coal above the hard coal gangue will lag behind. Therefore, the top coal will have poor caving characteristics. 


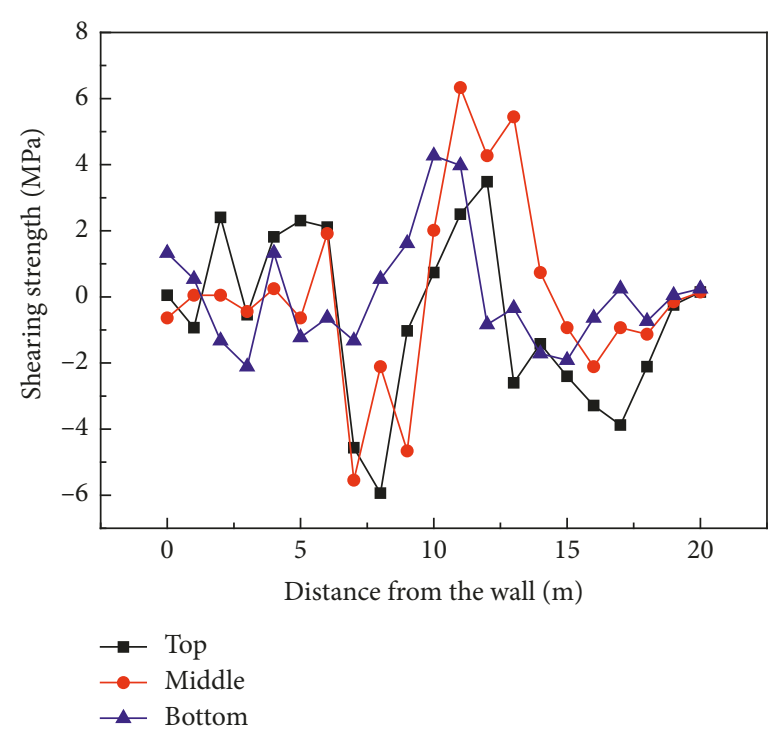

(a)

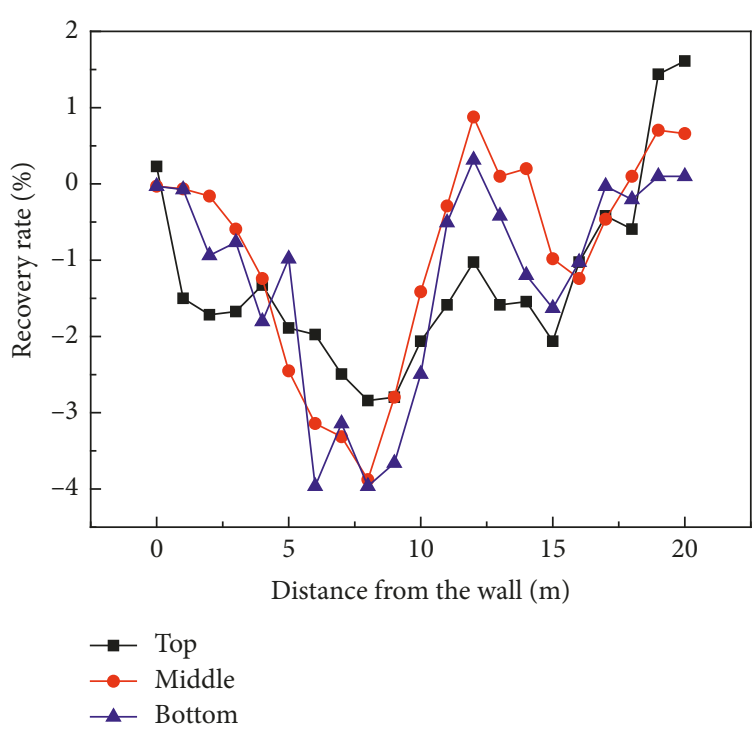

(b)

Figure 4: Distribution of shear stress in different layers of coal seam with $1.5 \mathrm{~m}$ thickness and different strength: (a) medium-strength and (b) high-strength hard coal gangue.

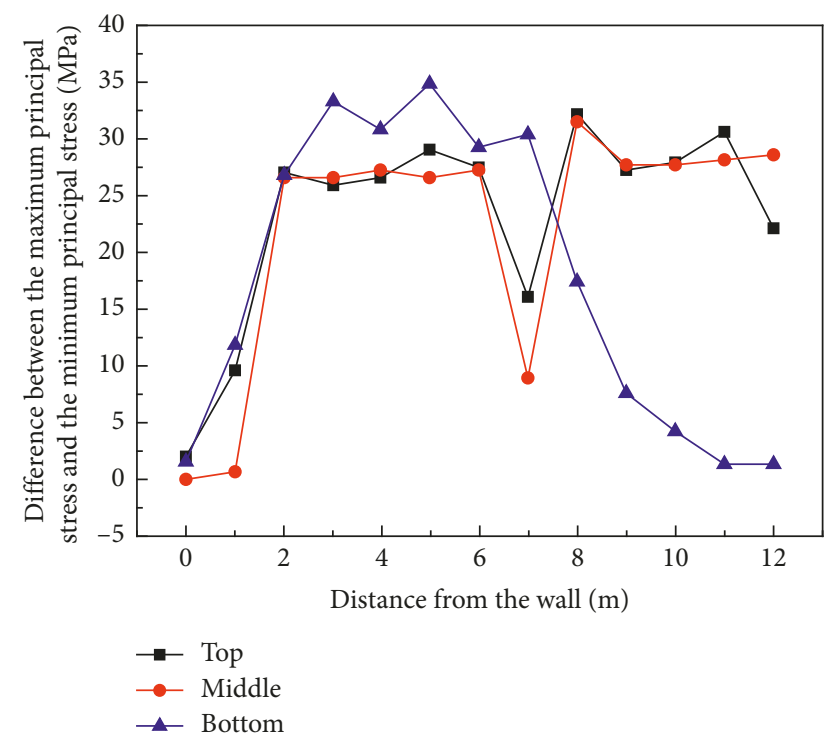

FIgURE 5: Curve of maximum and minimum principal stresses of different layers of $1.5 \mathrm{~m}$ thick medium-hard layer coal gangue.

A vertical displacement of $0.5 \mathrm{~m}$ thick highstrengthhard-coal coal gangue is shown in Figure 8. When the upper hard coal gangue is above the top control zone, the vertical displacement of each layer of top coal is larger and the difference is small. Similarly, while the top coal is broken fully, the existence of the upper hard coal gangue does not affect the release of the top coal. When the hard coal gangue is in the middle position, its vertical displacement with the upper coal is basically the same, which is obviously less than the vertical displacement of the lower coal, which indicates that the middle hard coal gang prevents the deformation of the upper coal. And it affects the flow of the upper top coal but does not affect the lower top coal.
The vertical displacements of the upper and lower top coal with different depths in the $1.5 \mathrm{~m}$ thick mediumstrength hard coal gangue are shown in Figure 9. As can be seen from that, with the increase of mining depth, the greater the vertical displacement of the top coal in the same layer, the greater the deformation of the top coal, the higher the destruction of the top coal, and the better the deployment of the top coal. The vertical deformation of the top coal in the top control area is greater than the top coal deformation in front of the coal wall because the deformation of the top coal in front of the coal wall is dominated by the horizontal displacement.

\section{Sparse Similarity Simulation}

4.1. Basic Principles and Methods. The dissociation model experiment combined with the site conditions of the coal mine, according to the similarity theory, designed the model with certain similarity ratio in the laboratory, measured the model data, and analyzed them.

Geological and production conditions of the N101 fully mechanized top coal caving face in Qinyuan coal mine: (1) the coal seam is a special thick coal seam, and the top coal thickness changes greatly; (2) the coal seam is directly topped with a mudstone coal and rock interbed with a thickness of $1.57 \mathrm{~m}$; the direct bottom is a mudstone with a thickness of $1.29 \mathrm{~m}$; the bottom of the coal seam, the coal seam, and the roof have a lower strength and is a "three soft" coal seam; (3) the structure of the coal seam is complex, and the soft and hard are different. The top coal in each layer of the coal seam is top-bottom coal (soft coal), top-middle top coal (hard coal), and medium top coal (soft coal) from top to bottom along the thickness direction. Middle and lower top coal (hard coal) and lower top coal (soft coal) are as shown in Figure 10; (4) the average coal thickness in the working face 


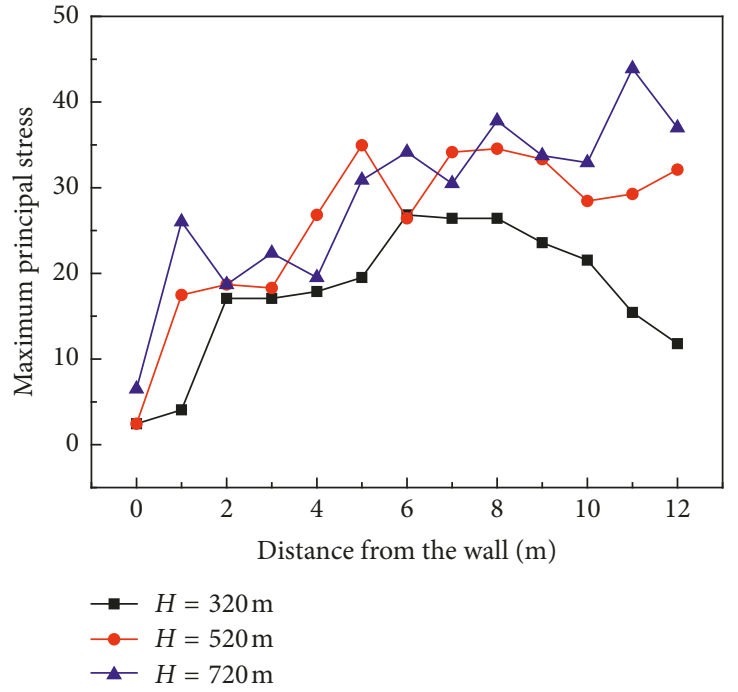

(a)

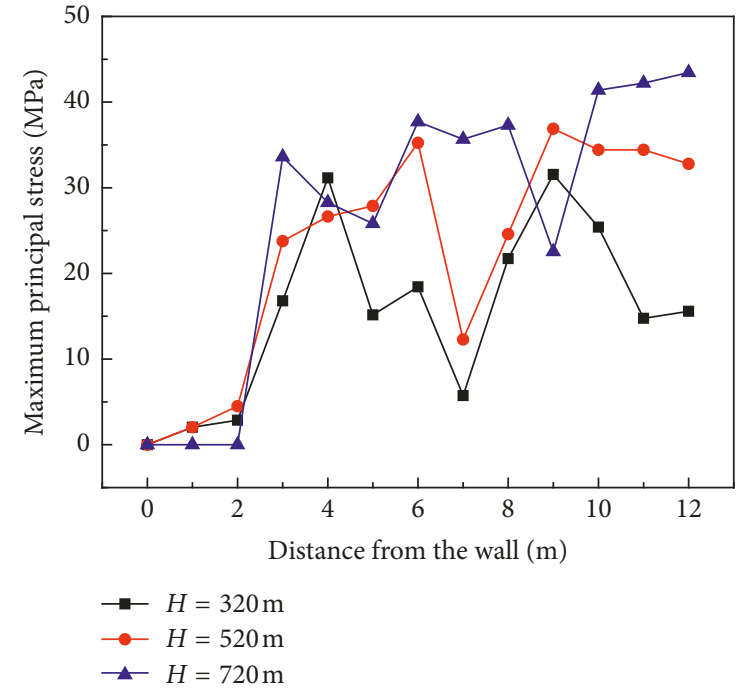

(b)

Figure 6: Maximum principal stress of (a) top coal and (b) lower coal with $1.5 \mathrm{~m}$ thick medium-strength hard coal gangue at different depths.

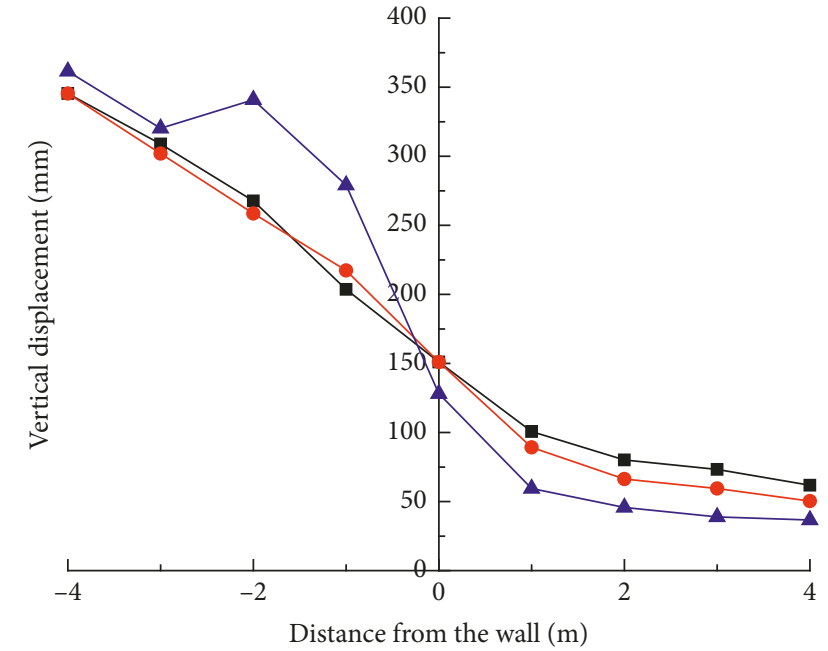

$$
\begin{aligned}
& -0.5 \mathrm{~m} \text { hard coal on top } \\
& -0.5 \mathrm{~m} \text { hard coal in middle } \\
& -0.5 \mathrm{~m} \text { hard coal at bottom }
\end{aligned}
$$

Figure 7: Vertical displacements of $0.5 \mathrm{~m}$ thick high-strength medium-strength coal seam and top coal.

is $8.7 \mathrm{~m}$, the mining height is $2.3 \mathrm{~m}$, and the top coal thickness is $6.4 \mathrm{~m}$; (5) work face bracket adopts ZF5500/17/ 28HQ-type anti-four-barlow-cutting top coal support.

In the mechanized top coal caving face, the formation of the top coal crushing block is the result of overburden rock bearing pressure, old roof rotation deformation, and repeated support of the support. The destruction of top coal is developed from the bottom-up. The lower coal is supported by pressure of the larger support. The degree of fragmentation above the support is smaller than that of the upper fragment. At the same time, due to the presence of highstrength hard top coal in coal seams, the fragmentation of

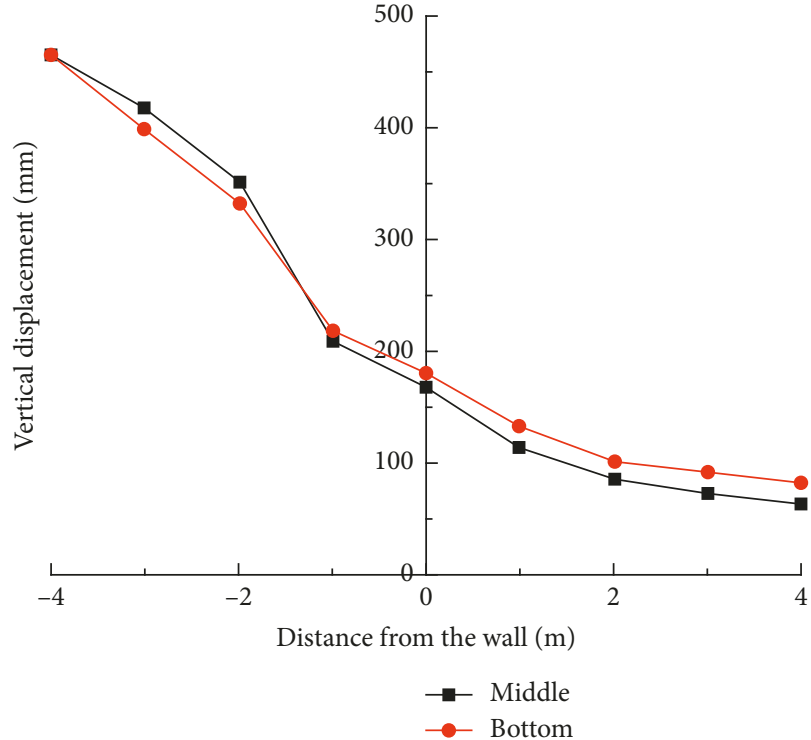

Figure 8: Vertical displacements of coal seams and top coal with $0.5 \mathrm{~m}$ high-strength upper hard layers.

hard coal seams is larger than that of soft coal seams, but at the same time, the fragmentation of soft coals at upper positions is larger than that of lower soft coals. Table 4 shows the top coal fragmentation at different levels measured on the site of the N101 fully mechanized top coal caving face.

4.2. Model Establishment. The frame size used in the experiment is $130 \mathrm{~cm}$ in length and $12 \mathrm{~cm}$ in width. The width of the top coal support used in the site is $1.5 \mathrm{~m}$. Considering the content of the study and the number of coal deposits per model, the width of the model frame is taken as the width of the two simulated supports in the model. That is, the width 


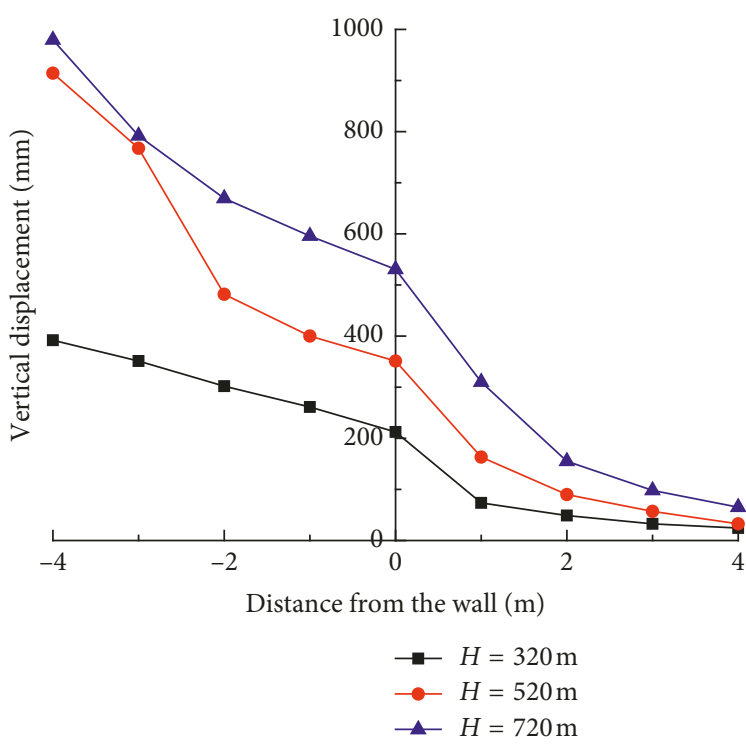

(a)

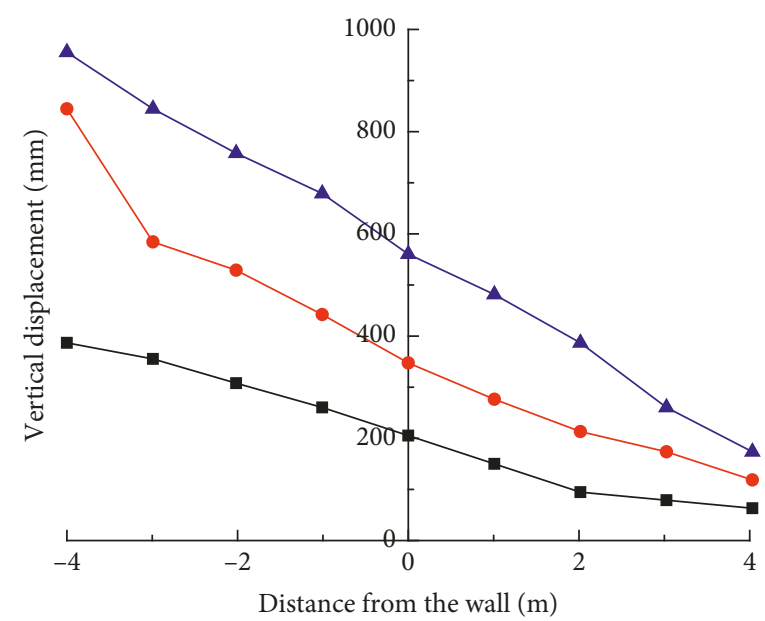

$\rightarrow H=320 \mathrm{~m}$
$\longrightarrow H=520 \mathrm{~m}$
$\longrightarrow H=720 \mathrm{~m}$

(b)

Figure 9: Vertical displacements of different layers of top coal with different thickness in $1.5 \mathrm{~m}$ thick medium-strength hard coal gangue. (a) Upper top coal. (b) Lower top coal.

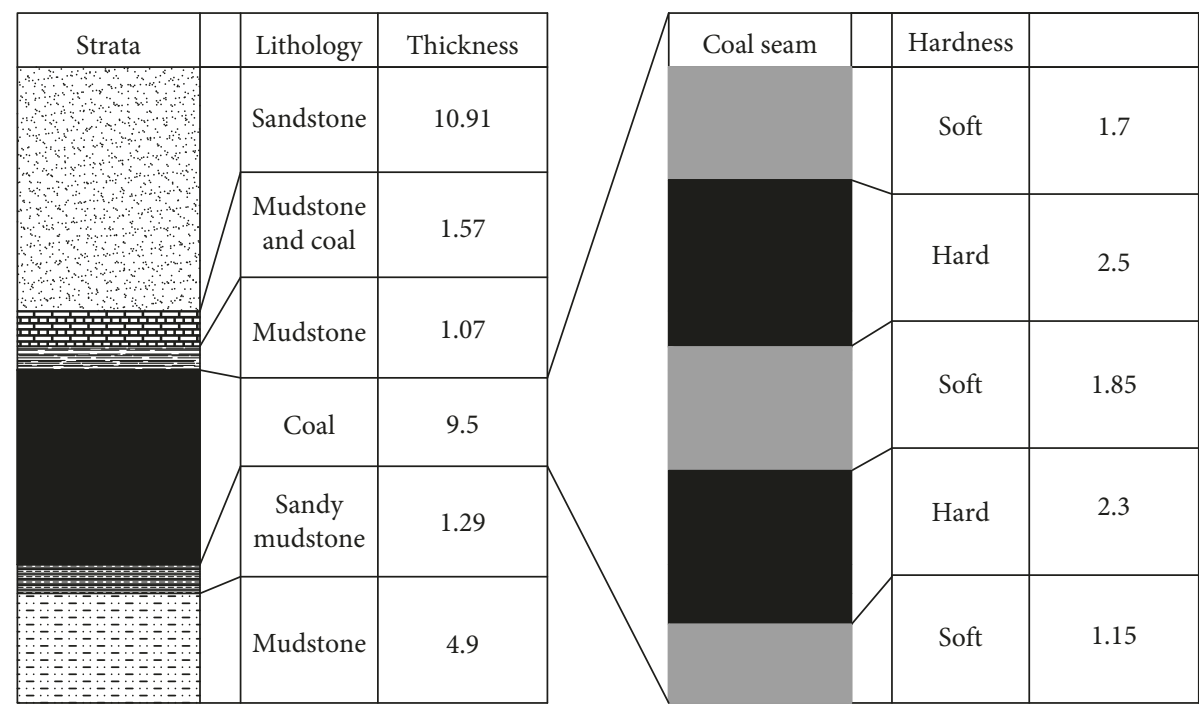

(a) (b)

FIgURE 10: (a) Coal seam comprehensive column shape at N101 fully mechanized caving face in Qinyuan coal mine. (b) Top coal schematic.

of the simulated support is $6 \mathrm{~cm}$. Therefore, the geometric similarity between the model and the prototype is $C_{1}=6 /$ $150=1: 25$.

Two angle steels are erected on the level of the coal opening, and a $2.4 \mathrm{~cm}$ wide steel bar is laid on it to simulate the shear depth of the coal face of the working face; in the model experiment, each stratified layer was laid horizontally, and gray coal with different particle sizes was selected for the top coal, and a thin black marker layer stone was laid between soft and hard top coal layers. The top and bottom layers of the direct roof are selected from different particle sizes and different color stones, respectively, red and white, as shown in Figure 11. Specific actual dimensions and model dimensions are shown in Tables 5 and 6, respectively.

In the model making process, the front of the model is covered with plexiglass, and the rear is fixed with layered angle steel. In order to simulate the on-site coal caving process, a special low-level caving coal support was used to simulate the actual propulsion and coal-discharging process. The weighted volume of the top coal and the direct roof in the model experiment was calculated in the natural state (see Table 7 for data).

During the experiment, $10 \mathrm{~cm}$ was left on each side of the model to eliminate the boundary effect, and taking into 
TABLe 4: Measured top coal block size.

\begin{tabular}{|c|c|c|c|c|c|c|}
\hline Horizon & $\begin{array}{l}\text { Measuring } \\
\text { point } 1\end{array}$ & $\begin{array}{l}\text { Measuring } \\
\text { point } 2\end{array}$ & $\begin{array}{l}\text { Measuring } \\
\text { point } 3\end{array}$ & $\begin{array}{l}\text { Measuring } \\
\text { point } 4\end{array}$ & $\begin{array}{l}\text { Measuring } \\
\text { point } 5\end{array}$ & $\begin{array}{l}\text { Measuring } \\
\text { point } 6\end{array}$ \\
\hline The lower top coal fragmental size $(\mathrm{cm})$ & 10 & 13 & 16 & 12 & 17 & 10 \\
\hline $\begin{array}{l}\text { The middle and lower top coal fragmental } \\
\text { size }(\mathrm{cm})\end{array}$ & 42 & 47 & 49 & 46 & 50 & 40 \\
\hline The middle top coal fragmental size $(\mathrm{cm})$ & 18 & 20 & 21 & 18 & 23 & 16 \\
\hline $\begin{array}{l}\text { The middle and upper top coal fragmental } \\
\text { size }(\mathrm{cm})\end{array}$ & 48 & 52 & 53 & 50 & 58 & 45 \\
\hline The upper top coal fragmental size $(\mathrm{cm})$ & 23 & 26 & 28 & 25 & 32 & 23 \\
\hline
\end{tabular}

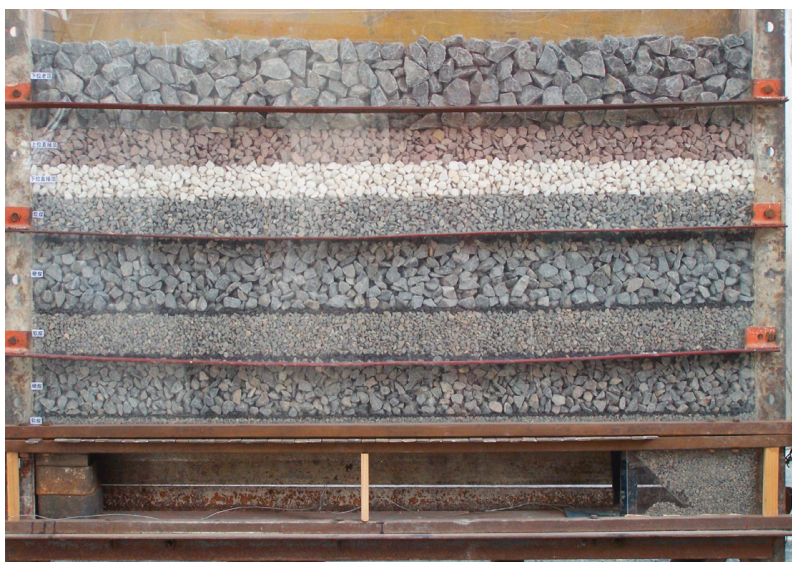

FIGURE 11: Overview of the experimental model of complex structure coal seam.

TABLe 5: Hydraulic support dimensions.

\begin{tabular}{|c|c|c|c|c|c|c|c|c|c|}
\hline \multicolumn{2}{|c|}{ Width } & \multicolumn{2}{|c|}{ Mining height } & \multicolumn{2}{|c|}{$\begin{array}{l}\text { Minimum control top } \\
\text { distance }\end{array}$} & \multicolumn{2}{|c|}{ Height of coal caving } & \multicolumn{2}{|c|}{$\begin{array}{l}\text { Cutting depth of coal } \\
\text { shearer }\end{array}$} \\
\hline Actual (m) & Model $(\mathrm{cm})$ & Actual (m) & $\operatorname{Mod}(\mathrm{cm})$ & Actual (m) & Model $(\mathrm{cm})$ & Actual (m) & Model $(\mathrm{cm})$ & Actual (m) & Model $(\mathrm{cm})$ \\
\hline 1.5 & 6 & 2.3 & 9.2 & 4.59 & 18.36 & 1.1 & 4.4 & 0.6 & 2.4 \\
\hline
\end{tabular}

TABLE 6: Experimental scheme for dissipation of top coal in coal seams with three soft and complex structures.

\begin{tabular}{|c|c|c|c|c|c|}
\hline \multirow{2}{*}{\multicolumn{2}{|c|}{ Horizon }} & \multicolumn{2}{|c|}{ Actual } & \multicolumn{2}{|c|}{ Model } \\
\hline & & Thickness (m) & Block degree $(\mathrm{mm})$ & Thickness $(\mathrm{cm})$ & Block degree $(\mathrm{mm})$ \\
\hline \multirow{5}{*}{ Top coal } & Bottom & 1.15 & 125 & 4.6 & 5 \\
\hline & Middle-bottom & 2.3 & 437.5 & 9.2 & 17.5 \\
\hline & Middle & 1.85 & 187.5 & 7.4 & 7.5 \\
\hline & Middle-top & 2.5 & 500 & 10 & 20 \\
\hline & Top & 1.7 & 250 & 6.8 & 10 \\
\hline \multirow{2}{*}{ Basic roof } & Bottom & 1.3 & 300 & 5.2 & 12.5 \\
\hline & Top & 1.3 & 375 & 5.2 & 15 \\
\hline Basic roof & Bottom & 3.637 & 1000 & 14.5 & 40 \\
\hline
\end{tabular}

account the control roof distance of the support, the effective length of the model coal drop test is $90 \mathrm{~cm}$, then the number of coal throwing for each experiment of three coal placement steps $=90 / 14.4=6$ times. Because three models of coal caving can be experimented on a single model, the experiment of the top coal emission law of the three-soft complex structure coal seam only needs to lay a model.
4.3. Experimental Procedure. The coal laying simulation process is as follows:

(1) Install a coal-spraying support, that is, place the support in the high-elevation space in the lower part of the model

(2) Install a baffle to seal the space behind the support (gob) 
TABLE 7: Parameters of top coal and direct roof.

\begin{tabular}{|c|c|c|c|c|}
\hline Horizon & Thickness $(\mathrm{cm})$ & Weighted volume $\left(\mathrm{cm}^{3} /\right.$ each $)$ & Sieving length $(\mathrm{mm})$ & $P\left(\mathrm{~g} / \mathrm{cm}^{3}\right)$ \\
\hline Bottom (soft) & 4.6 & 0.0301 & 5 & 1.403 \\
\hline Middle-bottom (hard) & 9.2 & 1.8182 & 17.5 & 1.352 \\
\hline Middle (soft) & 7.4 & 0.09901 & 7.5 & 1.357 \\
\hline Middle-top (hard) & 10 & 4.5333 & 20 & 1.367 \\
\hline Top (soft) & 6.8 & 0.4118 & 10 & 1.354 \\
\hline Bottom (white) & 5.2 & 0.9667 & 12.5 & 1.517 \\
\hline Top (red) & 5.2 & 1.3333 & 15 & 1.357 \\
\hline Bottom & 14.5 & 26.6667 & 40 & 1.493 \\
\hline
\end{tabular}

(3) Pullout the steel plate above the tail beam of the support and the position of the coal discharge port to allow the top coal to fall

(4) Open the bracket window and discharge the top coal

(5) While the enthalpy ratio is $1 \%$ (the ratio of vermiculite weight to the top coal weight above the coal discharge step), the window is closed

(6) Feed the coal support forward, and the shifting distance is a feeding distance of the coal mining machine

(7) Pullout a top-protecting coal plate above the support (two times for one time (6) (7); three times for one time (6) (7))

(8) Open the coal opening window to open the coal, when the yttrium content is $1 \%$ (the amount of vermiculite accounts for the total mass ratio of the top coal above the step), the window is closed; repeat the process from (6) to (8)

Using the electronic balance to weigh the coal and vermiculite weights, the top coal emission rate in the simulation experiment was obtained. The movement and emission patterns of top coal and scattered vermiculite were observed during the experiment. Analyze the top coal flow and release process in the continuous coal advancement of low coal placement and the coal waste separation line and flow boundary line in the top and bottom coal. Master the shape of the coal gangue flow field under different release technologies.

\subsection{Similar Simulation Results}

\subsubsection{Arch during the Top Coal Spill}

(1) Form of Arch. During the coal laying process, due to the randomness of the top coal shape and the relative position of the top coal block, the diversity of the form of arching occurs, and the arch is composed of coal blocks of soft and hard top coal. In the process of coal throwing, the arching types of coal boring are mainly the arching of the coal opening and the arching of the coal flow above the support tail beam.

(a) Arch opening: The opening of the coal opening is shown in Figure 12. The front arch foot rests on the tail beam of the support, and the rear arch foot falls on the coal flute on the border of the coal flow. With the continuous flow and release of top coal, the arch experienced a process of formation, expansion, and direct destruction; at the same time, the shape of the arches, the arc angles of the arch lines, and the constant changes in the spans (arch pitches) have also led to the formation of arches in various forms. In order to reduce the probability of top coal arching, the coal should swing as far as possible to increase the top coal recovery rate, and it can also be broken by arching, but this method will damage the scaffold.

(b) The soft and hard coal flows into the arch above the tail boom: During the process of coal laying, each layered top coal continuously shrinks in the direction toward the coal discharge port, and the soft coal body with the lower string speed and the relatively slow hard top coal body are mutually squeezed along the interface. Friction and uneven sinking result in the stress-balanced arch structure. This arch structure is formed at the interface between soft and hard top coal. As shown in Figure 13, the impact of the tail beam of the swing support on the arch structure is very small.

(2) Arch Probability. Experimental studies have shown that the probability of arching is the highest in the coal laying process when both are used. The probability of laying coal and arching under different coal laying steps is shown in Table 8. At the time of pick and place, an average of 0.43 coal arches were built at a time, up to a maximum of 3 . The average coal mining and arching were performed twice each time, which was 4.65 times that of one mining and one mining. The probability of arching is the lowest among three mining and one casting, and the average time for coal arching is 0.4 each time. The reason is that the span of the arch is large, the curvature is small, and it is difficult to form a stable arch structure.

\subsubsection{Charge Parameters}

(1) One Knife One Falling. At the time of one knife one falling (Figure 14), both the vermiculite and the top coal behind the low-level caving support move toward the coal opening, and the initial coal-bearing boundary line is a quadratic curve. Together with Figure 15, the speed of white and red gangue migration was greater than that of the middle-upper and 


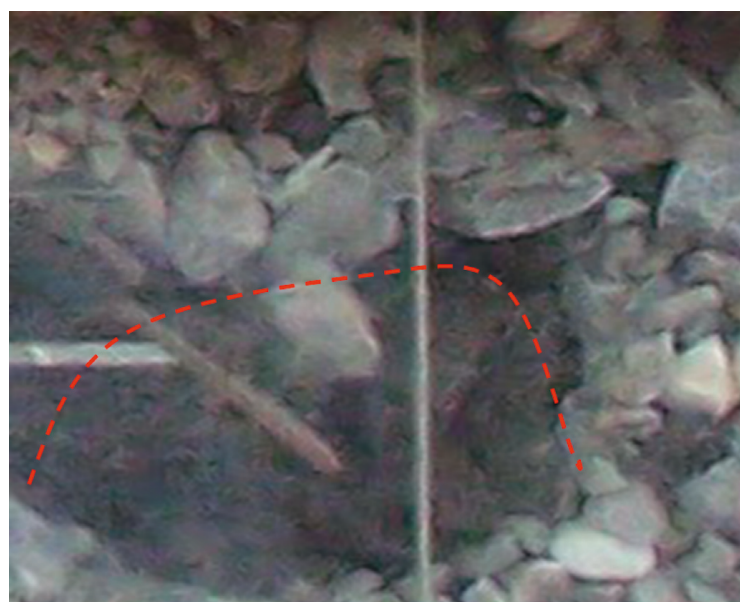

Figure 12: Arch opening.

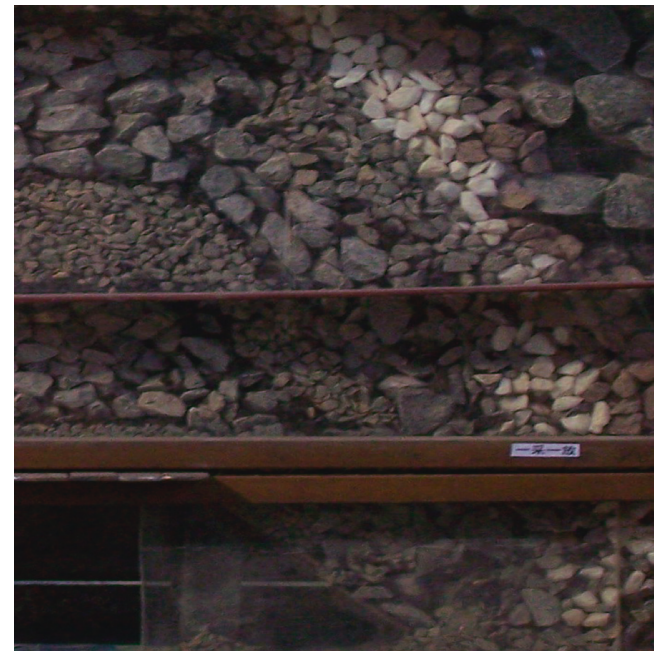

Figure 13: Arch during the flow of soft and hard coal.

middle-low hard top coals; before the hard top coal reached the coal opening, the average recovery rate of hard coal was $27.4 \%$, and the average recovery rate of soft coal was $55.2 \%$. Soft coal deposits have significantly more coal than hard coal. According to the data, the average recovery rate of top coal is $41.2 \%$, the lowest is $19.38 \%$, and the highest is $80.85 \%$, but the overall trend is upward. The recovery rate of top coal is far greater than that of thorium, and the average level of germanium is $3.6 \%$, obviously mixed. The coal deposits in the top, middle, and bottom top coal layers are significantly more than those in the lower, upper, and middle-upper layers; the amount of coal deposited in the upper-level top coal is the least.

(2) Two Knife One Falling. At the time of two knife one falling, the rear end of the support is a mixture of different pieces of top coal, the top coal is difficult to move, and the amount of coal reaching the coal opening is small, but the amount of coal discharged from the middle, lower, and middle layered top coal is obviously more than the lower, upper, middle, and top layered coal top coal discharge
(Figure 16). The starting point of the top coal movement is farther than that of a mining one, and the boundary line of the coal mine becomes slower and moves in parallel along the direction of advancement of the working face. The average recovery rate of top coal was $98.21 \%$, which was more than doubled from one mining experience to another, with the lowest being $59.66 \%$ and the highest being $182.72 \%$. The recovery rate of top coal is far greater than the rate of flooding, and the average level of flooding is $1.1 \%$. The rate of flooding is 2.5 percentage points lower than that of flooding. The average recovery rate of hard coal is $98.25 \%$, and the average recovery rate of soft coal is $98.16 \%$. The discharge rate of soft coal is basically the same as that of hard coal. See Figure 17 for details.

(3) Three Knife One Falling. At the time of three knife one falling, the coal-bearing boundary line moved forward but not smooth along the driving direction of the working face (Figure 18). The average recovery rate of top coal was $86.16 \%$, the lowest was $59.48 \%$, and the highest was $109.04 \%$. The recovery rate of top coal is about two times that of the one knife one falling, the top coal recovery rate is much higher than the flooding rate, the average level of the flooding rate is $1.1 \%$, and containing vermiculite rate is 2.5 percentage points lower than that of one knife one falling, and the mix is not obvious. The average recovery rate of hard coal is $83.88 \%$, and the average recovery rate of

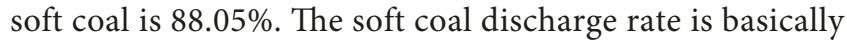
the same as the hard coal emission rate (see Figure 19 for details).

In the experiment, from seeing the complete discharge of the complex top coal, the amount of top coal discharged and the amount of rubble discharged were counted, and the ratio of the top coal recovery rate and the rate of containing germanium to the number of coal deposits during the coal release process is shown in Figure 20. During the first 13 coal deposits, the helium-containing rate was kept at or above $1 \%$, which was caused by manual operation errors. However, the overall trend of top coal recovery was reduced and the reduction was large. After the thirteenth coal deposit, the top coal recovery rate was stable at between $4 \%$ and $11 \%$, while the indention rate increased.

\section{Field Application Results and Discussion}

5.1. Working Face Overview. N101 face is located in the lower part of the northern mining area, the upper part of which is 303 working face (have been mined), the lower part is the unexplored area, the east is 102 working face, and the west is the unexcavated area. The elevation of the working surface is $520-471 \mathrm{~m}$, the elevation of the ground is 985$1043 \mathrm{~m}$, and the area of the working surface is $94990 \mathrm{~m}^{2}$.

The surface coal seam is dominated by bright coal and dark coal with more mirror coal and silk ribbons. The fissure is developed and the structure is complex. The bottom of the coal seam is $0.8-1.5 \mathrm{~m}$ in gray mudstone. The coal quality is poor, and the hardness coefficient is $f=1.6$. The coal seam is stable and is monoclinic, with a wavy $\mathrm{NE} 325^{\circ}$ and a coal seam inclination of $17-32^{\circ}$. It is $23^{\circ}$ and is close to the 
TABLE 8: Contrast ratio of arching at different coal caving distances.

\begin{tabular}{ccccc}
\hline Coal caving distance & One knife one falling & Two knife one falling & Three knife one falling \\
\hline \multirow{2}{*}{ The number of coal arching times each time } & Maximum & 3 & 6 & 2 \\
& Average & 0.43 & 2 & 0.4 \\
\hline
\end{tabular}

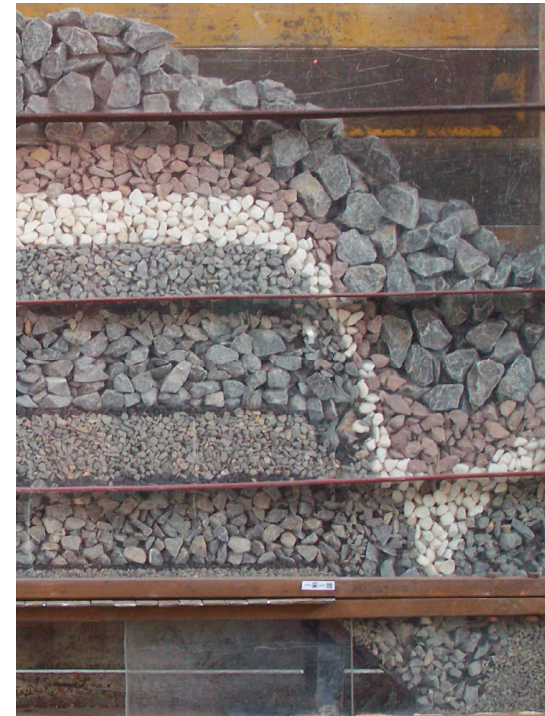

(a)

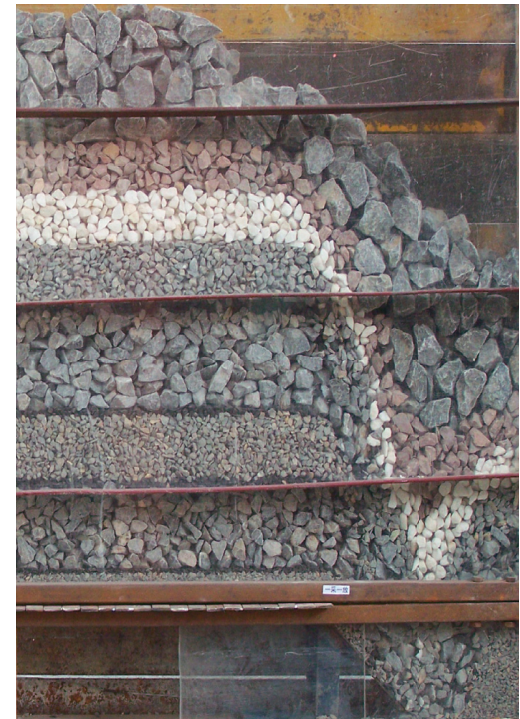

(b)

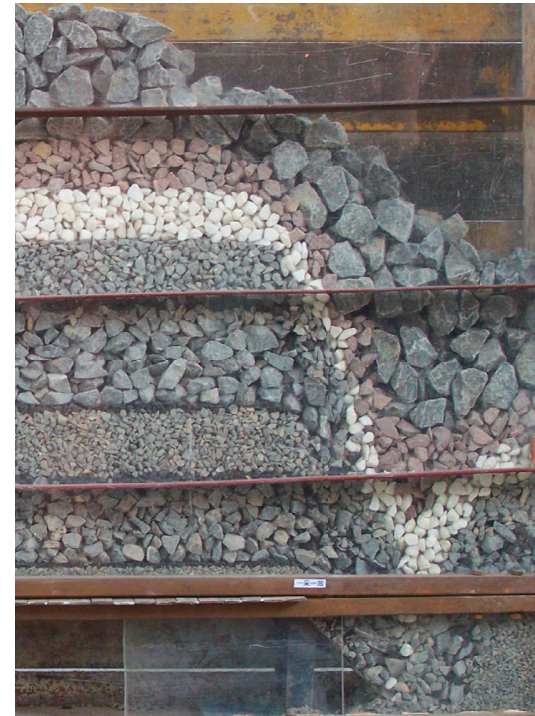

(c)

Figure 14: Dump top coal effect of one knife one falling. (a) Before relocation. (b) After relocation and before coal dumping. (c) After laying coal.

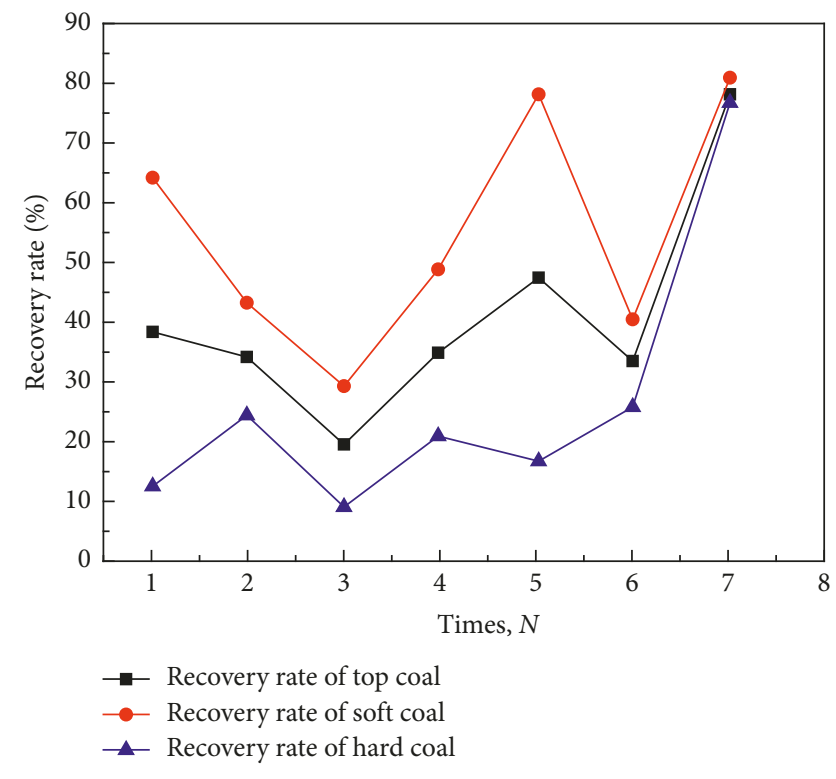

FIGURE 15: Top coal recovery ratio.

inclined coal seam. Coal seam roof and floor conditions are shown in Table 9. The working face mining method adopts the method of moving to the full-scale mining of the longwall fully mechanized top coal caving method. The coal cutting depth is $0.6 \mathrm{~m}$, the height of cutting coal is $2.3 \mathrm{~m}$, and the coal laying height is $6.4 \mathrm{~m}$, so the ratio of mining to cutting is $1: 2.78$. The coal discharge process adopts the operation mode of one knife one falling (putting coal in the step of $0.6 \mathrm{~m}$ ) and the picking and placing process is carried out in parallel.

\subsection{Mine Pressure Law}

5.2.1. Line Layout and Observation Method. $80 \mathrm{~m}$ ahead of the working face, in the upper slot along the side of the work face to play two drill holes, arranged in each hole five KSE-II1-type drill hole strain gauges, drill height is $1 \mathrm{~m}$ from the floor. The layout parameters of drilling and stress gauges in the working plane are shown in Figure 21.

The arrangement of measuring points on the working surface is shown in Figure 21. The 70\#, 42\#, 32\#, and 31\# brackets are arranged along the inclined direction of the working face to arrange the stations. Each front and rear column is installed with a round chart pressure selfrecording device on each diagonal line for continuous collection of record holders. The column cyclic resistance changes, including the initial support force, working resistance, and end-of-cycle resistance of the support.

5.2.2. Advance Bearing Pressure Size and Distribution. When drilling the strain gauge, the initial pressure is $10 \mathrm{MPa}$. After two days, the changes in the readings of various tables have stabilized. After stabilization, the distribution of lateral bearing pressure on the working face formed by roadway 


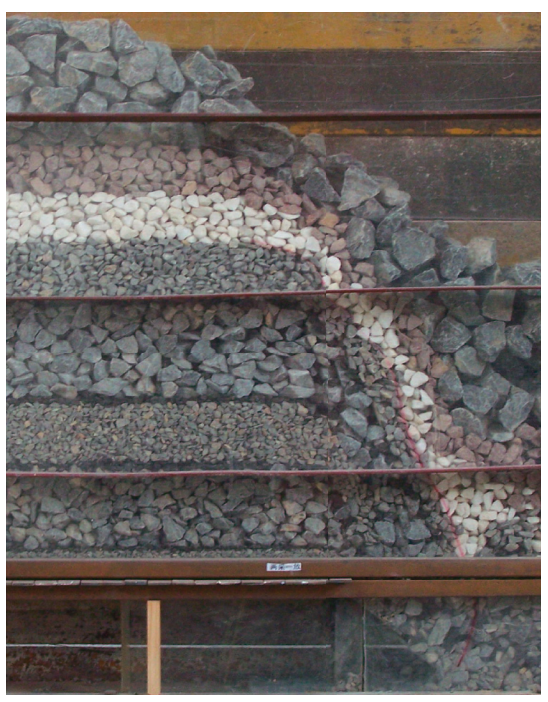

(a)

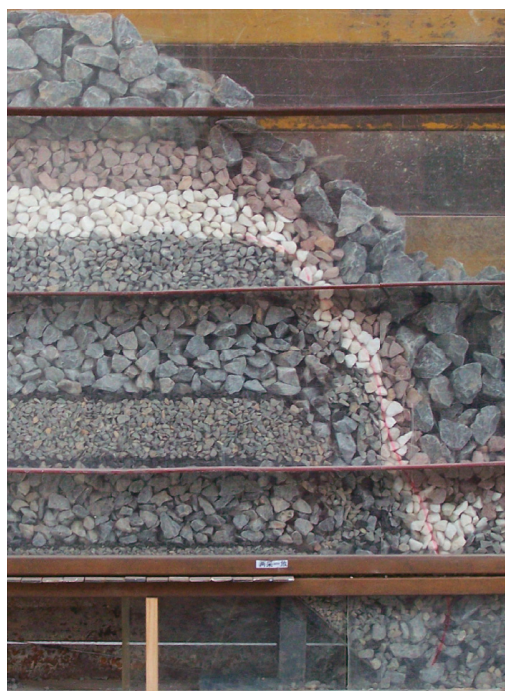

(b)

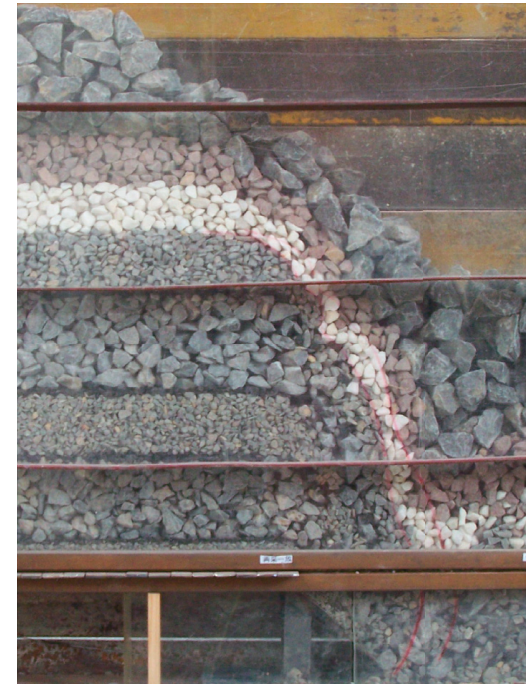

(c)

FIGURE 16: Dump top coal effect of two knife one falling. (a) Before relocation. (b) After relocation and before coal dumping. (c) After laying coal.

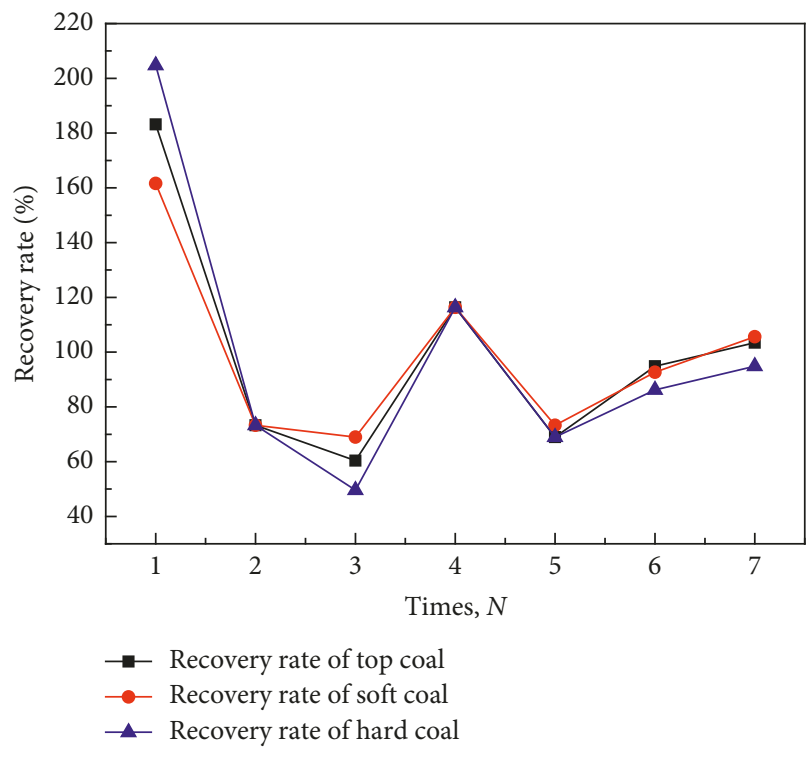

FIGURE 17: Recovery rates of two knife one falling.

digging can be obtained according to the readings of each table, as shown in Figure 22.

From Figure 22, the original rock stress at the measuring point is $9.5 \mathrm{MPa}$, the distance from the peak point to the roadway is about $6 \mathrm{~m}$, the lateral pressure influence range is about $9 \mathrm{~m}$, and the stress concentration factor of the lateral support pressure is 1.41 . When the measuring point distance is about $35 \mathrm{~m}$ away from the working surface, the counts of individual tables begin to change, indicating that the leading bearing pressure of the working face will begin to affect the stress at the measuring point.

The change in readings of the individual strain gauges along the face is shown in Figure 23. From Figure 23, it can be seen that the peak point of the leading bearing pressure is about $12 \mathrm{~m}$ from the working surface, and the influence range is about $35 \mathrm{~m}$. The stress concentration factor of the leading bearing pressure is 2.33 .

\subsubsection{Pressure Distribution in the Direction of the Inclined} Plane. Due to factors such as coal seam inclination, mining boundary conditions, mining process, coal and rock formation conditions, and support quality, the pressure on the longwall of the working surface may be different. Measurement and analysis of the pressure distribution of the working face in the direction of the face during the pressure period and during the nonpressure period is shown in Figure 24.

From Figure 24, we can see the pressure on the top surface of the work is relatively small during the period of pressure and during nonadvancement. During the pressure period, the pressure in the middle and lower parts is slightly greater than the pressure in the middle and upper parts; the pressure in the middle and lower parts during the nonpressure period is slightly lower than the pressure in the middle and upper parts.

\subsection{Top Coal Caving and Recovery of Fully Mechanized Caving} Face. Table 10 shows the statistical results of the coal throwing time of the support before and after the research of the project and the thickness of the same coal seam in the N101 fully mechanized top coal caving face. The statistical results of the coal discharge time show that the efficiency of coal caving on the face of the project before research is low, and the maximum coal-burning time is more than 20 minutes. On-site observations are mainly due to the high degree of fragmentation of hard coal in top coal, which results in large resistance and slow flow. Large hard coals are often blocked on the top of the shield cover beam or the coal opening, resulting in the deployment of top coal. It is reflected by the long coal releasing time. When the working 


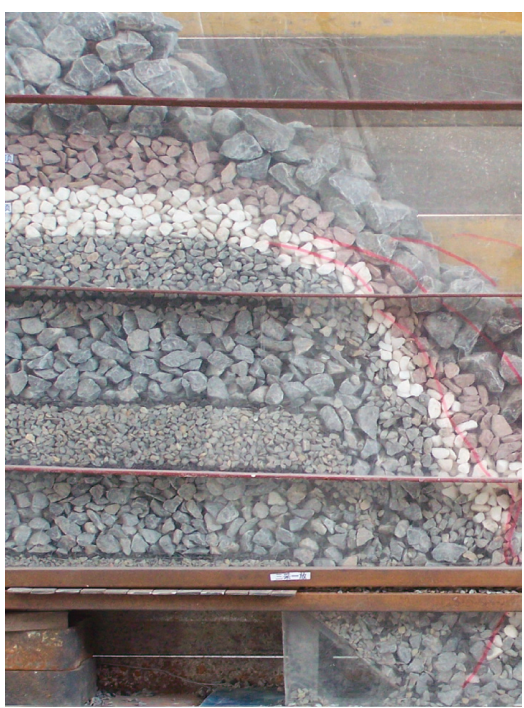

(a)

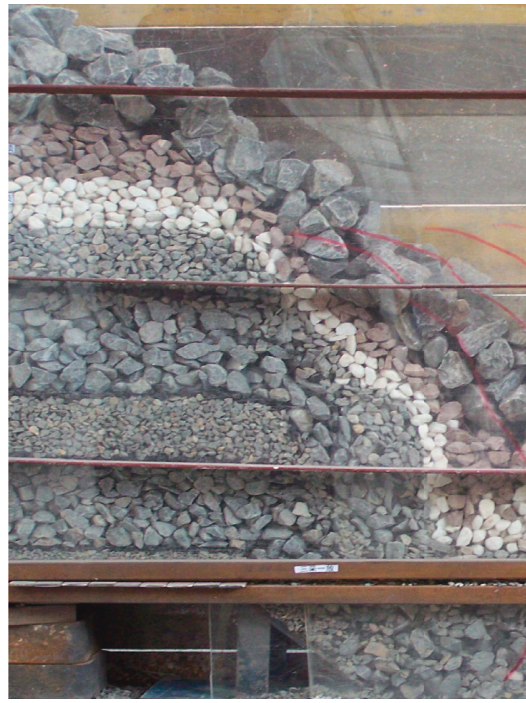

(b)

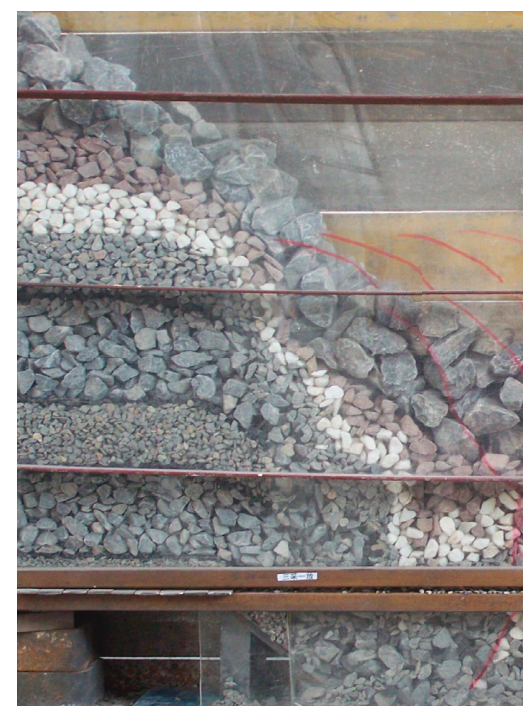

(c)

FIGURE 18: Dump top coal effect of three knife one falling. (a) Before relocation. (b) After relocation and before coal dumping. (c) After laying coal.

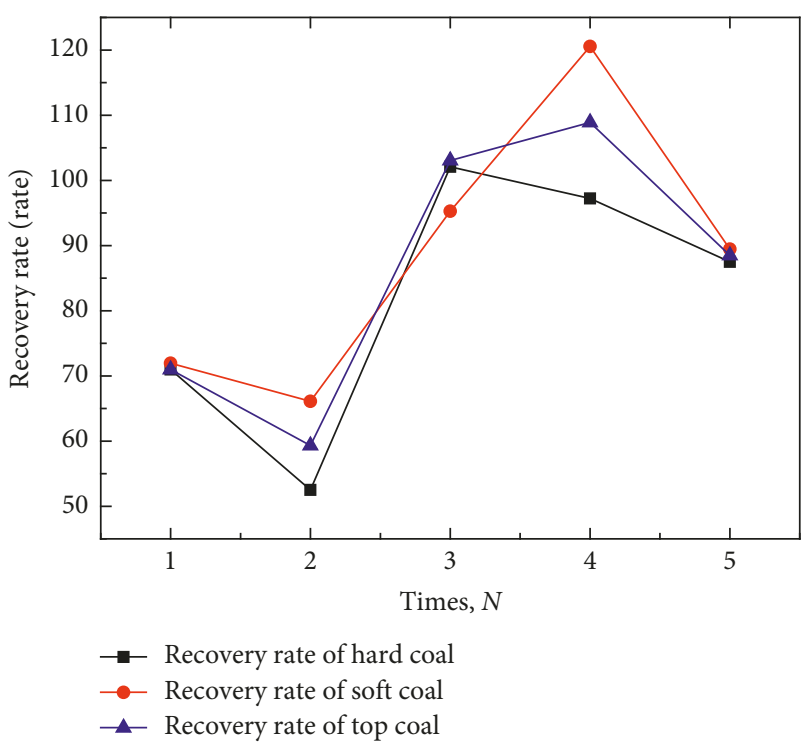

Figure 19: Recovery rates of three knife one falling.

face was blasted through a deep hole, the released coal was small in size and loosely broken. The frequency of blocking the coal opening at the large coal block was significantly reduced, and the top coal was allowed to settle smoothly, which represented a shortening of the coal discharge time. The release of coal has been improved [40, 41].

During the research of the project, while continuing to adopt the coal mining and coal mining steps, they also adopted a series of technical measures to improve the deployment of top coal in complex structures. Drilling holes are arranged along the two forward slots in the advance face, and then deep-hole blasting is performed to destroy the integrity of the hard coal in the top coal and cause vibration cracks to be fully broken. Then, in the gap between the brackets, drills were drilled with a coal-powered electric drill,

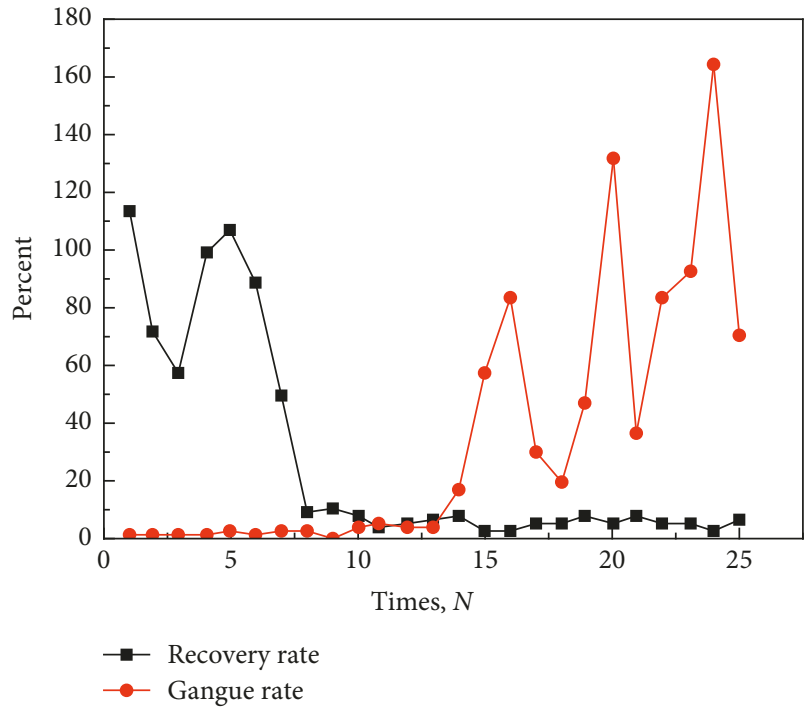

FIgURE 20: Top coal recovery rates and containing vermiculite rate of three knife one falling.

and the cannons were placed so that the top coal further destroyed the fragmentation and the top coal was ejected. The top coal recovery rate at the fully mechanized top coal caving face has been significantly improved. See Figure 25. During the research period of the project, the average recovery rate of the fully mechanized top coal caving face was $86.9 \%$, and the maximum recovery rate was $90.1 \%$. The fully mechanized top coal caving face was highly productive and achieved significant technical and economic benefits.

\section{Conclusion}

In this paper, based on a comprehensive analysis of the current status of research on thick coal seams with complex 
TABle 9: Coal seam roof and bottom conditions on N101 working face.

\begin{tabular}{lccc}
\hline Roof and bottom name & Rock name & Thickness $(\mathrm{m})$ & Lithologic characteristics \\
\hline $\begin{array}{l}\text { Basic roof } \\
\text { Immediate roof }\end{array}$ & Sandstone & 10.91 & $\begin{array}{c}\text { Gray siltstone, deep gray mudstone with gray and } \\
\text { white coarse sand layer }\end{array}$ \\
$\begin{array}{l}\text { False roof } \\
\text { Mirect bottom }\end{array}$ & Mudstone coal rock interbedding & 1.57 & $\begin{array}{c}\text { Grayish white coarse sandstone with dark mudstone, } \\
\text { muddy cement, loose }\end{array}$ \\
Previous bottom & $\begin{array}{c}\text { Mudstone } \\
\text { Mudstone }\end{array}$ & 1.07 & $\begin{array}{c}\text { Dark gray, gray-black mudstone with a thin layer of } \\
\text { mudstone in the lower middle } \\
\text { Black thick mudstone }\end{array}$ \\
\hline
\end{tabular}

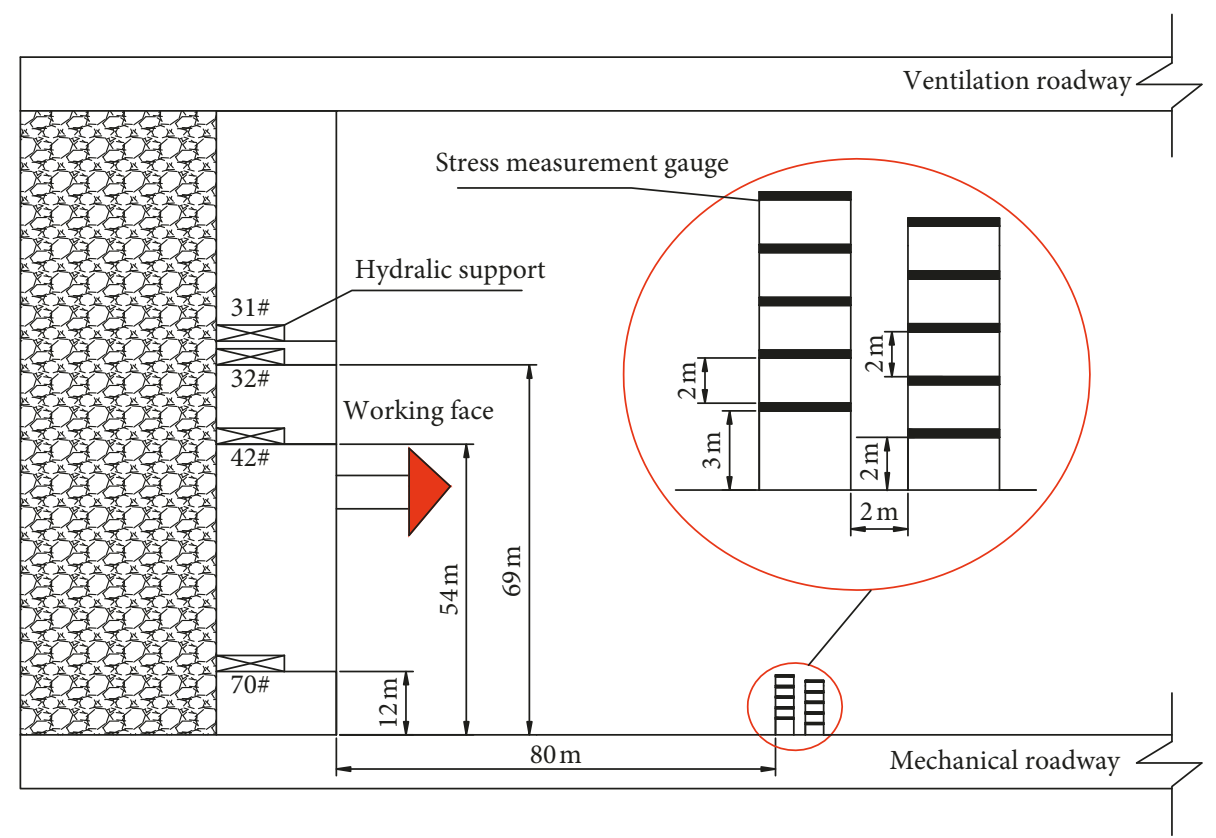

FIGURE 21: Drilling stress measurement point layout and measuring point layout.

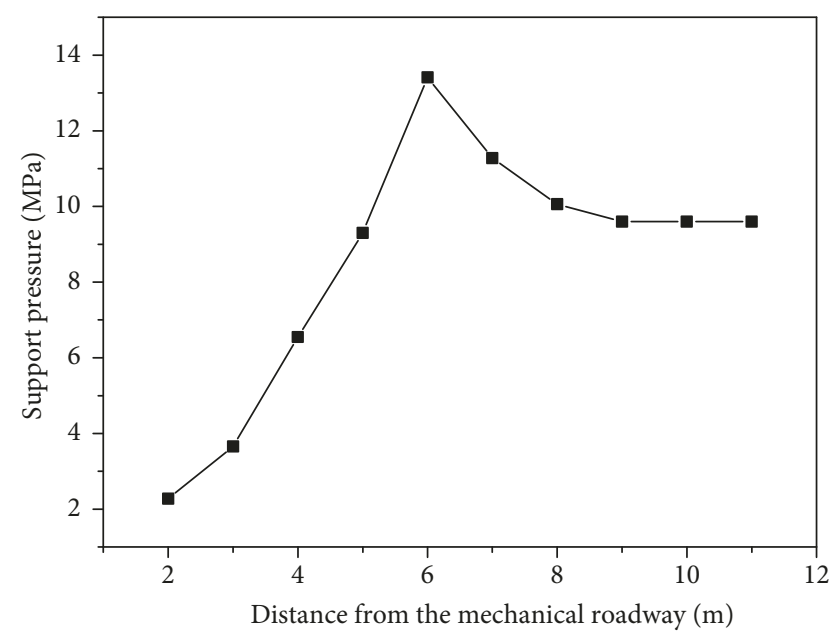

FIGURE 22: Lateral bearing pressure distribution on working face.

structures at home and abroad, theoretical analysis, similar laboratory simulations, and numerical simulations are used to study the rules of the top coal burst in the fully mechanized top coal caving mining in complex thick coal seams.

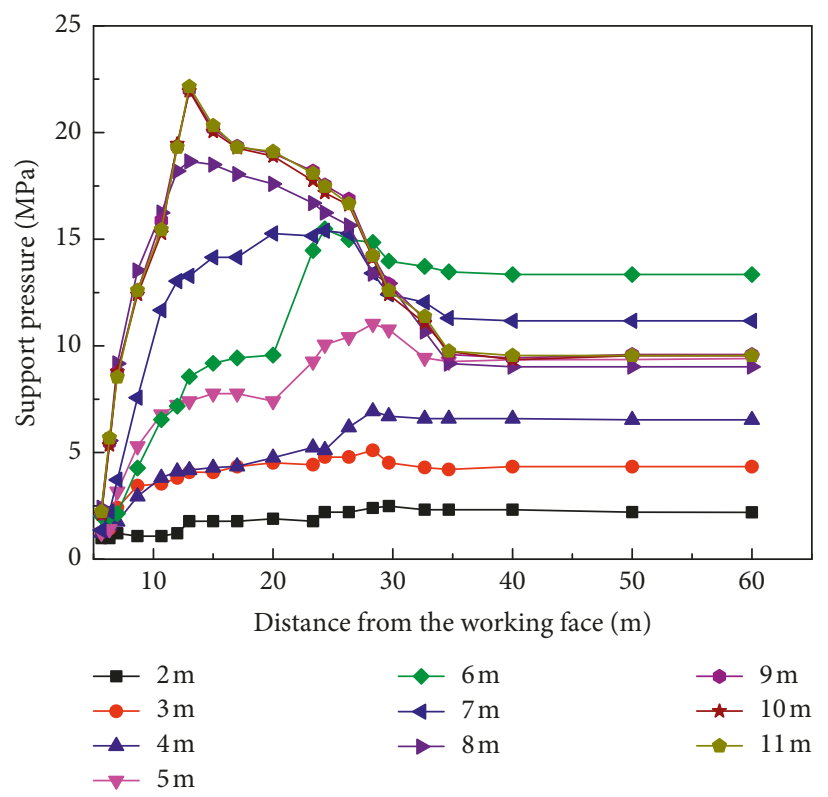

FIGURE 23: Distribution of advance bearing pressure at different measuring points in boreholes. 


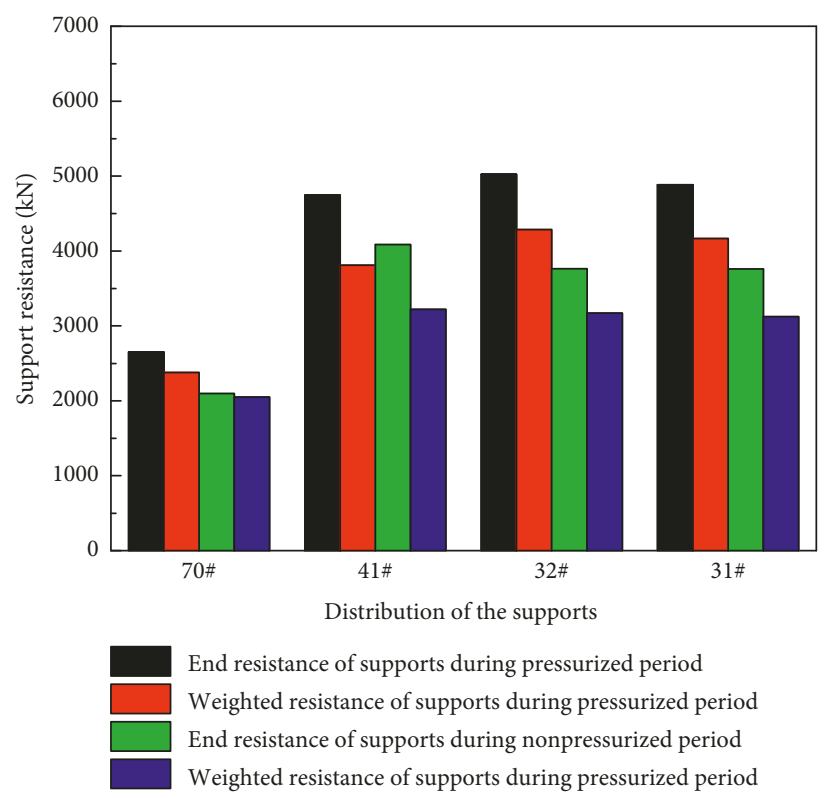

FIGURE 24: Longitudinal resistance distribution of working face during pressure period and nonpressure period.

TABle 10: Statistics on the caving time.

\begin{tabular}{|c|c|c|c|c|c|}
\hline \multicolumn{6}{|c|}{ The caving time of hydraulic support (s) } \\
\hline Maximum time & $\begin{array}{l}\text { Minimum time before } \\
\text { beginning of subject }\end{array}$ & $\begin{array}{l}\text { Average } \\
\text { time }\end{array}$ & Maximum time & Minimum time during subject & Average time \\
\hline 1286 & 197 & 742 & 312 & 48 & 180 \\
\hline
\end{tabular}

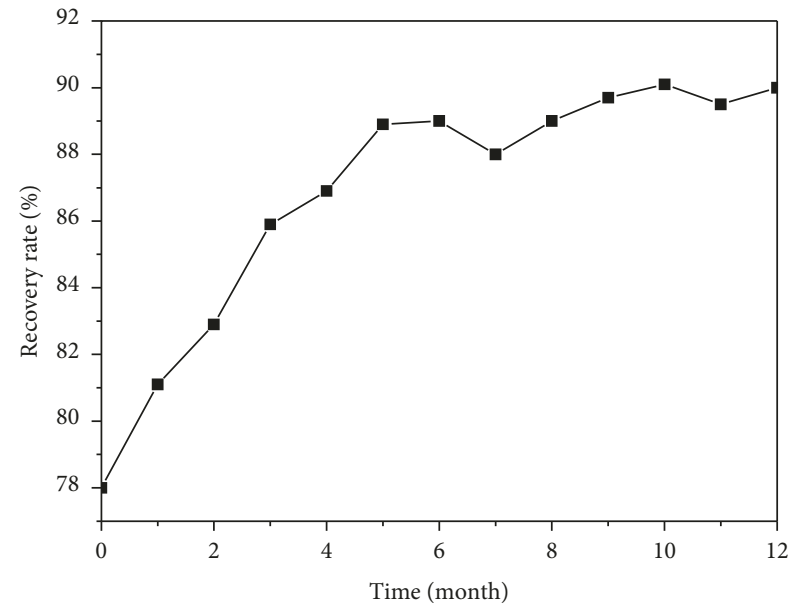

FIGURE 25: Recovery rate of working face during the study period.

The coal deposit process parameters were analyzed and studied. Combined with the geology and production technology conditions of the N101 fully mechanized coal mining face in Qinyuan coal mine, the field application and actual measurement analysis were performed, and the following main conclusions were obtained:

(1) Through establishing the mechanical model of breaking the complex structure, it is found that the strength limit of the hard coal gangue and the coal body in the top coal body determines the top coal caving characteristics. After the top coal is destroyed by strength, the continued destruction of the top coal depends on the increase of top coal deformation. With the increasing of the thickness of the hard coal gangue in the top coal, or the strength of the hard coal gangue, the top coal as a whole gradually exhibits the fracture characteristics of medium or hard top coal. As the plastic zone locates in the top control zone, the cumulative displacement of the top coal is low, the loose zone is reduced, the loose expansion range formed by the top coal is small, the expansion deformation of the top coal is small, the plastic flow time is short, and the top coal is poorly discharged. If the thickness of the hard coal gangue in the top coal or the strength of the hard coal gangue is reduced, the plastic zone is located in front of the coal wall.

(2) Through theoretical analysis, the formula for the elastic rupture stress of the hard layer coal gangue medium reaching the limit state failure is obtained. The elastic fracture stress of hard coal gangue increases with the increasing of unidirectional compressive strength and lateral stress of hard coal gangue. The breaking factor formulas of hard coal and soft coal are introduced, and the crushing degree of top coal is described by the size of rupture factor. The larger the rupture factor is, the better the cracking effect of top coal is. 
(3) Through numerical simulation, it is found that, with the decreasing of the thickness or strength of hard coal gangue in the top coal, the peak pressure of support pressure is flat and far away from the coal wall. In the midlevel hard coal gangue condition, the top coal below the hard coal gangue is smoothly and quickly released along with the shift frame, and the middle hard layer coal gangue forms the delamination, the intermediate hard gangue coal gangue breaks insufficiently, and the broken lumps are large. Because the lower speed of the hard coal gangue block is slower than that of the top coal, it will hinder the top coal body, and the top coal above the hard coal gangue will lag behind. Therefore, the top coal has poor caving characteristics. With the increasing of the depth of top coal in different layers, the maximum principal stress also increases, and the top coal is increasingly broken. With the increasing of mining depth, the top coal plastic zone has become larger, and the top coal has become more destructive.

(4) Through similar simulation experiments, it was found that, due to the randomness of the top coal shape and the relative position of the top coal block in the coal caving process, the diversity of the arch formation was caused, and the arch was composed of coal blocks of soft and hard top coal. This phenomenon hindered the deployment of top coal. Experimental results show that the probability of arching is greatest in the process of two knife one falling. At the time of one knife one falling, an average of 0.43 coal arches was built at a time, up to a maximum of 3 . At the time of two knife one falling, an average of 3 coal arches was built at a time, which was 4.65 times that of one knife one falling. The probability of arching is the lowest among two knife one falling, and the average time for coal arching is 0.4 each time. The reason is that the span of the arch is large, the curvature is small, and it is difficult to form a stable arch structure.

(5) According to the on-site experiments, it was found that the top coal recovery rate was the highest when the top coal caving step of the complex structure is adopted for two knife one falling mining operations. In view of the characteristics of top coal crushing in fully mechanized coal seams with complex structure, a coal mining measure to reduce the fragmentation of top coal is proposed, namely, preblasting of top coal by loosening, improving the external environment of the coal body, and hydraulic fracturing of top coal.

\section{Data Availability}

The data used to support the findings of this study are available from the corresponding author upon request.

\section{Conflicts of Interest}

The authors declare that they have no conflicts of interest.

\section{Authors' Contributions}

Xuelong Li conducted the field measurements and wrote the manuscript. All authors gave final approval for publication.

\section{Acknowledgments}

This work was supported by the National Science and Technology Major Project of China (Grant no. 2016ZX05043005), State Key Research Development Program of China (Grant no. 2016YFC0801404), and National Natural Science Foundation of China (Grant no. 51674050), which are gratefully acknowledged. First of all, we would like to thank Professor Qingting Hu of State Key Laboratory of Coal Mine Disaster Dynamics and Control; he provided us with some equipment for field measurement. Second, we are grateful to Yongjiang Luo, who provided comments that substantially improved the manuscript.

\section{References}

[1] X. Liu, D. Song, X. He, Z. Wang, M. Zeng, and K. Zeng, "Nanopore structure of deep-burial coals explored by AFM," Fuel, vol. 246, pp. 9-17, 2019.

[2] G. Si, S. Jamnikar, J. Lazar et al., "Monitoring and modelling of gas dynamics in multi-level longwall top coal caving of ultra-thick coal seams, part I: borehole measurements and a conceptual model for gas emission zones," International Journal of Coal Geology, vol. 144-145, pp. 98-110, 2015.

[3] E. Y. Wang, X. F. Liu, X. Q. He, and Z. H. Li, "Acoustic emission and electromagnetic radiation synchronized monitoring technology and early-warning application for coal and rock dynamic disaster," Journal of China University of Mining and Technology, vol. 47, no. 5, pp. 953-959, 2018.

[4] Q. Zou and B. Lin, "Fluid-solid coupling characteristics of gasbearing coal subjected to hydraulic slotting: an experimental investigation," Energy and Fuels, vol. 32, no. 2, pp. 1047-1060, 2018.

[5] L. Q. Duan, L. Dong, and L. J. Ma, "Experimental study of acoustic emission characteristics of foamed concrete under uniaxial compression," Journal of China University of Mining and Technology, vol. 47, no. 4, pp. 742-747, 2018.

[6] S.-h. Tu, Y. Yong, Y. Zhen, X.-t. Ma, and W. Qi, "Research situation and prospect of fully mechanized mining technology in thick coal seams in China," Procedia Earth and Planetary Science, vol. 1, no. 1, pp. 35-40, 2009.

[7] J. Wang, "Development and prospect on fully mechanized mining in Chinese coal mines," International Journal of Coal Science and Technology, vol. 1, no. 3, pp. 253-260, 2014.

[8] J. Xu, N. Wang, and Y. Wang, "Multi-pyramid image spatial structure based on coarse-to-fine pyramid and scale space," CAAI Transactions on Intelligence Technology, vol. 3, no. 4, pp. 228-234, 2018.

[9] H. Alehossein and B. A. Poulsen, "Stress analysis of longwall top coal caving," International Journal of Rock Mechanics and Mining Sciences, vol. 47, no. 1, pp. 30-41, 2010.

[10] X. Li, E. Wang, Z. Li, Z. Liu, D. Song, and L. Qiu, "Rock burst monitoring by integrated microseismic and electromagnetic radiation methods," Rock Mechanics and Rock Engineering, vol. 49, no. 11, pp. 4393-4406, 2016.

[11] X. Li, Z. Li, E. Wang et al., "Pattern Recognition of Mine Microseismic and Blasting Events Based on Wave Fractal Features," Fractals, vol. 26, no. 3, article 1850029, 2018. 
[12] L. Yu, S. H. Yan, H. Y. Yu, and Z. Zhang, "Studying of dynamic bear characteristics and adaptability of support in top coal caving with great mining height," Procedia Engineering, vol. 26, pp. 640-646, 2011.

[13] D. Szurgacz and J. Brodny, "Dynamic tests of a leg in a powered roof support equipped with an innovative hydraulic system," E3S Web of Conferences, vol. 41, p. 03019, 2018.

[14] D. Szurgacz and J. Brodny, "Analysis of rock mass dynamic impact influence on the operation of a powered roof support control system," E3S Web of Conferences, vol. 29, p. 00006, 2018.

[15] D. Szurgacz and J. Brodny, "Analysis of load of a powered roof support's hydraulic leg," E3S Web of Conferences, vol. 71, p. 00002, 2018.

[16] W. Guo, Y. Tan, and E. Bai, “Top coal caving mining technique in thick coal seam beneath the earth dam," International Journal of Mining Science and Technology, vol. 27, no. 1, pp. 165-170, 2017.

[17] X. R. Bai and J. M. Li, Modern Caving Mining Theories and Practical Technology, China University of Mining Techonology Press, Xuzhou, China, 2001.

[18] J. Zhang, Z. Zhao, and Y. Gao, "Research on top coal caving technique in steep and extra-thick coal seam," Procedia Earth and Planetary Science, vol. 2, pp. 145-149, 2011.

[19] J. Wang and Z. Wang, "Systematic principles of surrounding rock control in longwall mining within thick coal seams," International Journal of Mining Science and Technology, vol. 29, no. 1, pp. 65-71, 2019.

[20] D. W. Mckee, S. J. Clement, J. Almutairi, and J. Xu, "Survey of advances and challenges in intelligent autonomy for distributed cyber-physical systems," CAAI Transactions on Intelligence Technology, vol. 3, no. 2, pp. 75-82, 2018.

[21] S. J. Xu, X. P. Lai, and F. Cui, "Top coal flows in an excavation disturbed zone of high section top coal caving of an extremely steep and thick seam," Mining Science and Technology, vol. 21, no. 1, pp. 99-105, 2011.

[22] S. Yang, J. Zhang, Y. Chen, and Z. Song, "Effect of upward angle on the drawing mechanism in longwall top-coal caving mining," International Journal of Rock Mechanics and Mining Sciences, vol. 85, pp. 92-101, 2016.

[23] R. Singh and T. N. Singh, "Investigation into the behaviour of a support system and roof strata during sublevel caving of a thick coal seam," Geotechnical and Geological Engineering, vol. 17, no. 1, pp. 21-35, 1999.

[24] N. E. Yasitli and B. Unver, "3-D numerical modelling of stresses around a longwall panel with top coal caving," Journal of the Southern African Institute of Mining and Metallurgy, vol. 105, no. 5, pp. 287-300, 2005.

[25] H. Xie and H. W. Zhou, "Application of fractal theory to topcoal caving," Chaos, Solitons and Fractals, vol. 36, no. 4, pp. 797-807, 2008.

[26] W.-1. Shen, J.-b. Bai, W.-f. Li, and X.-y. Wang, "Prediction of relative displacement for entry roof with weak plane under the effect of mining abutment stress," Tunnelling and Underground Space Technology, vol. 71, pp. 309-317, 2018.

[27] W. Wang, G. Zhao, G. Lou, and S. Wang, "Height of fractured zone inside overlying strata under high-intensity mining in China," International Journal of Mining Science and Technology, vol. 29, no. 1, pp. 45-49, 2019.

[28] B. Kong, Z. Li, E. Wang, W. Lu, L. Chen, and G. Lu, "experimental study for characterization the process of coal oxidation and spontaneous combustion by electromagnetic radiation technique," Process Safety and Environmental Protection, vol. 119, pp. 285-294, 2018.
[29] G. Cheng, T. Ma, C. Tang, H. Liu, and S. Wang, “A zoning model for coal mining-induced strata movement based on microseismic monitoring," International Journal of Rock Mechanics and Mining Sciences, vol. 94, pp. 123-138, 2017.

[30] R. S. Yang, Y. L. Zhu, X. L. Zhu, D. M. Guo, and G. H. Li, "Discussions on some security mining problems of fullymechanized top coal mining in "three soft" large inclined angle working face," Procedia Engineering, vol. 26, pp. 1144-1149, 2011.

[31] X. Z. Xie and T. L. Zhao, "Analysis on the top-coal caving structure of extra-thick hard coal seam with shallow depth in fully mechanized sublevel caving mining," Journal of China Coal Society, vol. 41, no. 2, pp. 359-366, 2016.

[32] N. Zhang and C. Liu, "Arch structure effect of the coal gangue flow of the fully mechanized caving in special thick coal seam and its impact on the loss of top coal," International Journal of Mining Science and Technology, vol. 26, no. 4, pp. 593-599, 2016.

[33] X. Kong, E. Wang, S. Hu, R. Shen, X. Li, and T. Shen, "Fractal characteristics and acoustic emission of coal containing methane in triaxial compression failure," Journal of Applied geophysics, vol. 124, pp. 139-147, 2016.

[34] B. Hakan, O. Ferid, and A. Osman, "Prediction of the stresses around main and tail gates during top coal caving by $3 \mathrm{D}$ numerical analysis," International Journal of Rock Mechanics and Mining Sciences, vol. 76, pp. 88-97, 2015.

[35] D. Yun, Z. Liu, W. Cheng, Z. Fan, D. Wang, and Y. Zhang, "Monitoring strata behavior due to multi-slicing top coal caving longwall mining in steeply dipping extra thick coal seam," International Journal of Mining Science and Technology, vol. 27, no. 1, pp. 179-184, 2017.

[36] A. Vakili and B. K. Hebblewhite, "A new cavability assessment criterion for longwall top coal caving," International Journal of Rock Mechanics and Mining Sciences, vol. 47, no. 8, pp. 1317-1329, 2010.

[37] S. J. Feng, S. G. Sun, Y. G. Lv, and J. Lv, "Research on the height of water flowing fractured zone of fully mechanized caving mining in extra-thick coal seam," Procedia Engineering, vol. 26, pp. 466-471, 2011.

[38] Z. H. Chen, H. P. Xie, and Z. M. Lin, "Study on falling ability of top coal during top coal caving by damage mechanics," Chinese Journal of Rock Mechanics and Engineering, vol. 21, no. 8, pp. 1136-1140, 2002.

[39] W. J. Wang, C. Q. Zhu, and R. Q. Xiong, “An artificial neural network for distinguishing the difficulty degree of roof coal caving of steep seam," Journal of China Coal Society, vol. 27, no. 2, pp. 134-138, 2002.

[40] J. Wang, B. Yu, H. Kang et al., "Key technologies and equipment for a fully mechanized top-coal caving operation with a large mining height at ultra-thick coal seams," International Journal of Coal Science and Technology, vol. 2, no. 2, pp. 97-161, 2015.

[41] B. Jiang, "Behaviors of overlying strata in extra-thick coal seams using top-coal caving method," Journal of Rock Mechanics and Geotechnical Engineering, vol. 8, no. 2, pp. 238247, 2016. 


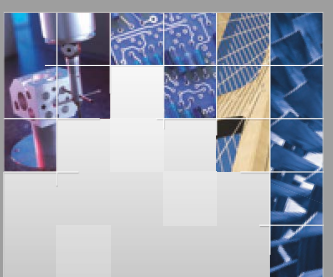

\section{Enfincering}
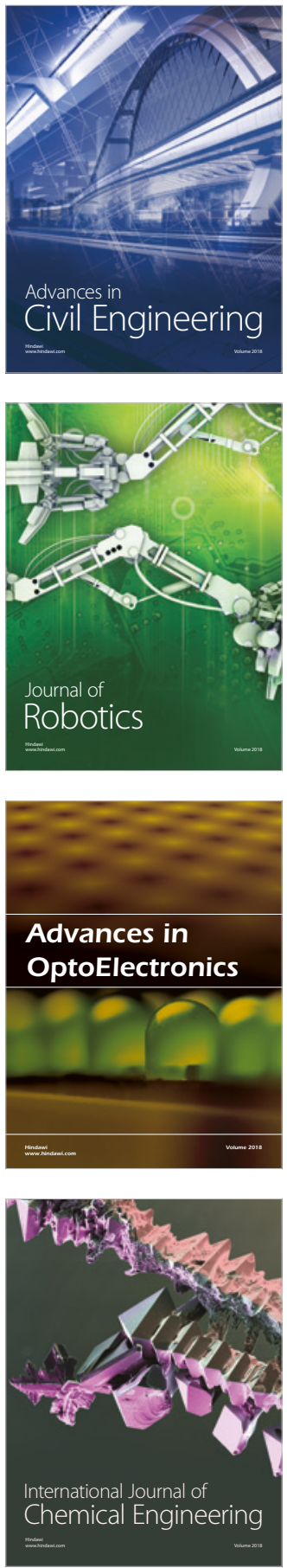

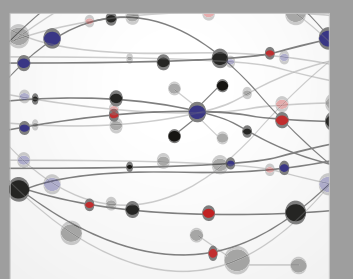

\section{Rotating \\ Machinery}

The Scientific World Journal

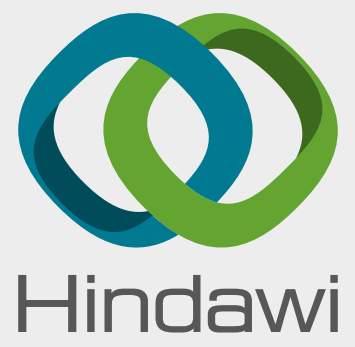

Submit your manuscripts at

www.hindawi.com
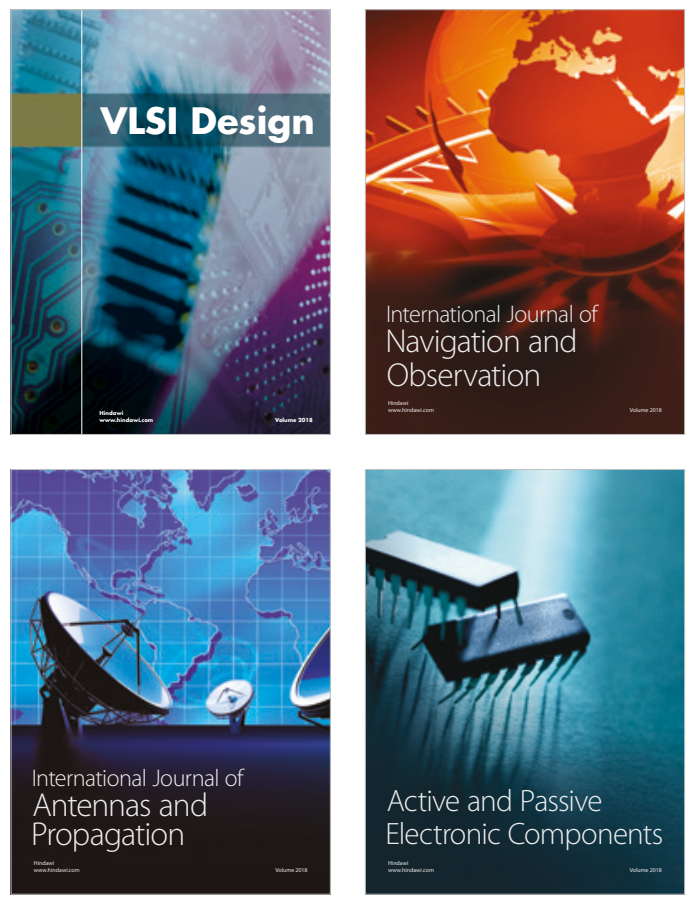
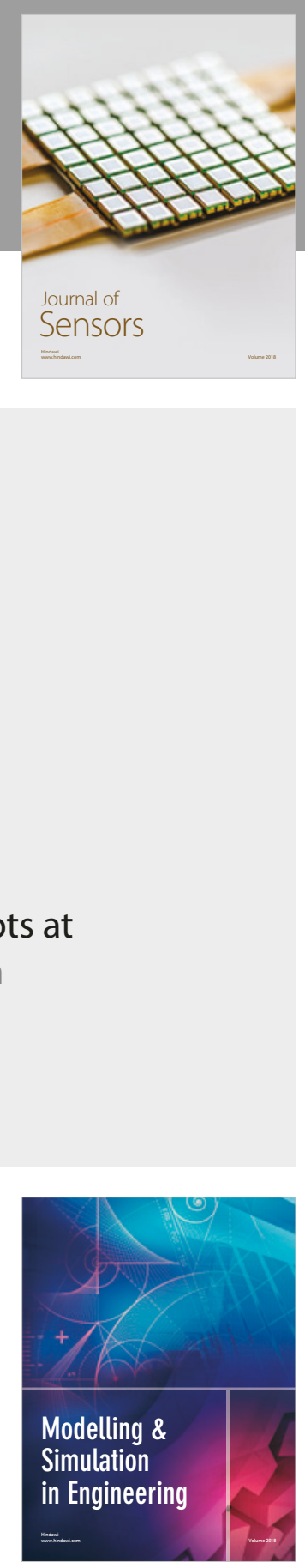

\section{Advances \\ Multimedia}
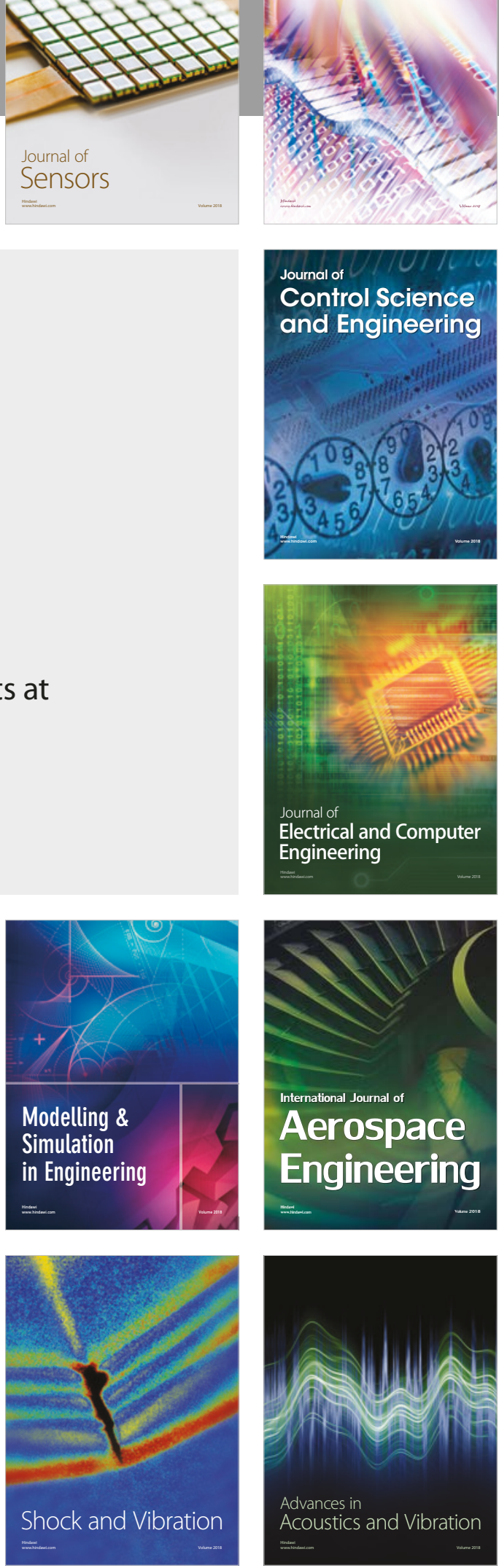